\title{
Regularized 13-moment equations: shock structure calculations and comparison to Burnett models
}

\author{
By M. TORRILHON ${ }^{1}$ AND H. STRUCHTRUP \\ ${ }^{1}$ ETH Zurich, Seminar for Applied Mathematics, ETH Zentrum, CH-8092 Zürich, Switzerland \\ manuel@math.ethz.ch \\ ${ }^{2}$ University of Victoria, Department of Mechanical Engineering, Victoria BC, V8W3P6, Canada \\ struchtr@me.uvic.ca
}

(Received 8 October 2002 and in revised form 23 April 2004)

Recently a new system of field equations for the accurate description of flows in rarefied gases, called regularized 13-moment equations, was obtained by means of a hybrid gas kinetic approach. The first part of this paper discusses the relationship of the new system to classical high-order theories like the Burnett and super-Burnett equations as well as to modified models like the augmented and regularized Burnett equations. In the second part, shock structure calculations with the new theory are presented and compared to direct-simulation Monte Carlo (DSMC) solutions and to solutions of the Burnett models. Owing to additional higher-order dissipation in the system, the profiles are smooth for any Mach number, in contrast to the results of Grad's 13-moment case. The results show reliable quantitative agreement with DSMC simulations for Mach numbers up to $M_{0} \approx 3.0$. The agreement is better for Maxwell molecules than for hard spheres. The results of the augmented Burnett equations are comparable, but these equations are shown to be spatially unstable. Additionally, a validiation procedure for the new equations is presented by investigating the positivity of Grad's distribution function.

\section{Introduction}

The regularized 13-moment equations (R13) have been obtained recently in Struchtrup \& Torrilhon (2003). The purpose of the new equations is to describe the flow of rarefied gases more accurately than classical theories, e.g. the system of Navier-Stokes and Fourier (NSF). The derivation is based on the original 13moment equations of Grad and a Chapman-Enskog-like expansion around a nonequilibrium. Hence, the new theory combines the two fundamental approaches to the Boltzmann equation: Grad's moment method, Grad (1949), and ChapmanEnskog expansion, Chapman \& Cowling (1970). Within the framework of kinetic theory, several extensions to the NSF theory have been derived by means of the original Chapman-Enskog expansion around the equilibrium, yielding the Burnett and super-Burnett equations, Chapman \& Cowling (1970), Shavaliyev (1993). However, these model suffer from inherent linear instabilities, see Bobylev (1982). Hence, several corrections or modifications have been proposed in order to remedy the instabilities. Two prominent examples are the augmented Burnett equations of Zhong, MacCormack \& Chapman (1991, 1993), and the regularized Burnett equations of Jin \& Slemrod (2001) and Jin, Pareschi \& Slemrod (2002). 
In Struchtrup \& Torrilhon (2003) it has been shown that the regularized 13-moment equations contain the Burnett and super-Burnett equations in an asymptotic manner, i.e. the Burnett and super-Burnett relations appear if the R13-system is expanded in terms of the Knudsen number by means of a Chapman-Enskog expansion. However, in contrast to the Burnett and super-Burnett equations, the R13-system is linearly stable for all wavelengths and frequencies. While in Struchtrup \& Torrilhon (2003) the correspondence of R13 and super-Burnett equations was shown only for the linear case, we extend the result in this paper to the nonlinear, one-dimensional case in $\S 2.2$. Additionally, we give a detailed discussion of the augmented and the regularized Burnett equations and compare these models to the regularized 13-moment equations in $\S \S 2.3$ and 2.4. Both modified Burnett models suffer from instabilities or inconsistencies which are overcome with the R13-system.

After the discussion of the equations, this paper presents shock structure solutions obtained from the regularized 13-moment equations. In a shock wave a gas experiences a fast transition between two equilibrium states across a domain of only few mean free paths. Owing to the absence of boundary effects and because of its essential one-dimensionality, the normal shock wave is one of the simplest flows with large deviations from thermodynamical equilibrium. Hence, the shock structure became a popular and challenging benchmark problem for testing theoretical descriptions of non-equilibrium and rarefied flows.

Fundamental measurements of complete shock structures have been accurately conducted, e.g. in Alsmeyer (1976) or Schmidt (1969), and furnish data with which to compare theoretical results. The experiments have been satisfactorily reproduced in calculations with the direct-simulation Monte-Carlo method (DSMC) (Bird 1998). However, so far no rational continuum description of rarefied flow has succeeded in predicting the experimental evidence accurately over a significant range of the shock wave Mach number $M_{0}$. Classical theories like the NSF system predict the shock thickness (see §6) at least qualitatively (Gilberg \& Paolucci 1953), but the quantitative agreement is restricted to Mach numbers below $M_{0} \approx 1.3$. Even worse, the NSF theory cannot even reproduce trends for the shock asymmetry (see also §6), as is shown e.g. in Alsmeyer (1976), Pham-Van-Diep, Erwin \& Muntz (1991) and $\mathrm{Au}$ (2001). These results reflect the fact that the NSF equations are restricted to near-equilibrium flows.

The shock structure profiles of the R13-system are compared to the results of Burnett models as well as to solutions of the direct-simulation Monte-Carlo method. From the comparison with DSMC profiles, we conclude that the new theory gives quantitatively reliable results up to a Mach number $M_{0} \approx 3.0$, especially for Maxwell molecules. Beyond that number at least qualitative features of the shock waves are captured. The Burnett and super-Burnett equations introduce strong oscillations into the solutions for shock structures, see e.g. Uribe, Velasco \& Garcia-Colin (1998) and Zhong et al. (1993) and $\S 5$ below. The augmented Burnett equations, though unstable in space, yield acceptable solutions with only small oscillations. The profiles are compared to DSMC and to the solutions of the R13-system.

The moment method proposed in Grad $(1949,1958)$ also attempts to approach the shock wave problem. Grad's 13-moment case, however, describes the shock thickness accurately only up to $M_{0} \approx 1.1$, and furthermore the profiles are spoiled by subshocks beyond $M_{0}=1.65$, see Grad (1952). Nevertheless, the general moment method of Grad is capable of describing shock structures accurately, provided sufficiently many moments are considered, see $\mathrm{Au}$ (2001), Au, Torrilhon \& Weiss (2001), Müller \& Ruggeri (1998), Weiss (1995). In the new regularized 13-moment equations the classical 
equations of Grad for 13-moments are included as a special limit (Struchtrup \& Torrilhon 2003). However, as will be seen in $\S 4.1$, the problem of subshocks is resolved in the regularized theory. Thus a major disadvantage of Grad's 13-moment case, non-smooth shock structures, is overcome.

The paper is organized as follows: The next section introduces the new regularized 13-moment equations, discusses the various expansions and compares the system to the augmented and the regularized Burnett equations. In $\S 3$ we present equations suitable for shock structure calculations and some remarks concerning the numerical method are given. Then, the general behaviour of the solutions of the regularized 13-moment equations, like smoothing of subshocks, is discussed. A comparison to DSMC profiles together with solutions of the augmented Burnett equations are given in $\S 5$, while in $\S 6$ we present the results for classical quantities derived from shock profiles like shock thickness and asymmetry. In the last section we consider the positivity of the distribution function in order to obtain a validity condition.

\section{Regularized 13-moment equations}

\subsection{The R13 equations}

The regularized 13-moment (R13) equations for monatomic gases were derived in Struchtrup \& Torrilhon (2003), and here we just present the results. The R13 equations are a set of field equations for the 13 variables $\rho_{A}=\left\{\varrho, \varrho v_{i}, \varrho \varepsilon=\frac{3}{2} \varrho R T, \sigma_{i j}, q_{i}\right\}$, where $\varrho$ is the mass density, $v_{i}$ is the gas velocity, $\varepsilon$ is the specific internal energy, $T$ is the temperature, $R$ is the specific gas constant, $\sigma_{i j}$ is the trace-free part of the pressure tensor, and $q_{i}$ is the heat flux vector. The field equations for these variables are the conservation laws for mass, momentum and energy,

$$
\left.\begin{array}{l}
\frac{\partial \rho}{\partial t}+\frac{\partial \varrho v_{k}}{\partial x_{k}}=0, \\
\frac{\partial \rho v_{i}}{\partial t}+\frac{\partial}{\partial x_{k}}\left(\rho v_{i} v_{k}+p \delta_{i k}+\sigma_{i k}\right)=0, \\
\frac{\partial}{\partial t}\left(\rho \varepsilon+\frac{1}{2} \rho v_{i}^{2}\right)+\frac{\partial}{\partial x_{k}}\left(\rho \varepsilon v_{k}+\frac{1}{2} \rho v_{i}^{2} v_{k}+p v_{k}+\sigma_{i k} v_{i}+q_{k}\right)=0,
\end{array}\right\}
$$

plus additional field equations for the stress deviator

$$
\frac{\partial \sigma_{i j}}{\partial t}+\frac{\partial \sigma_{i j} v_{k}}{\partial x_{k}}+\frac{4}{5} \frac{\partial q_{\langle i}}{\partial x_{j\rangle}}+2 p \frac{\partial v_{\langle i}}{\partial x_{j\rangle}}+2 \sigma_{k\langle i} \frac{\partial v_{j\rangle}}{\partial x_{k}}+\frac{\partial m_{i j k}}{\partial x_{k}}=-\frac{p}{\mu} \sigma_{i j},
$$

and heat flux

$$
\begin{gathered}
\frac{\partial q_{i}}{\partial t}+\frac{\partial q_{i} v_{k}}{\partial x_{k}}+\frac{5}{2} p R \frac{\partial T}{\partial x_{i}}+\frac{5}{2} \sigma_{i k} R \frac{\partial T}{\partial x_{k}}+R T \frac{\partial \sigma_{i k}}{\partial x_{k}}-\sigma_{i k} R T \frac{\partial \ln \varrho}{\partial x_{k}}-\frac{\sigma_{i j}}{\varrho} \frac{\partial \sigma_{j k}}{\partial x_{k}} \\
+\frac{7}{5} q_{k} \frac{\partial v_{i}}{\partial x_{k}}+\frac{2}{5} q_{k} \frac{\partial v_{k}}{\partial x_{i}}+\frac{2}{5} q_{i} \frac{\partial v_{k}}{\partial x_{k}}+\frac{1}{2} \frac{\partial R_{i k}}{\partial x_{k}}+\frac{1}{6} \frac{\partial \Delta}{\partial x_{i}}+m_{i j k} \frac{\partial v_{j}}{\partial x_{k}}=-\frac{2}{3} \frac{p}{\mu} q_{i} .
\end{gathered}
$$

Here, $p=\varrho R T$ is the pressure, and $\mu$ denotes the viscosity. Indices in angular brackets denote the symmetric trace-free parts of tensors. The above equations contain the additional quantities $m_{i j k}, R_{i k}, \Delta$, and constitutive equations are required to close the equations. With the choice

$$
m_{i j k}=R_{i k}=\Delta=0
$$


the above set of equations is reduced to the well-known set of 13-moment equations of Grad $(1949,1958)$. In Struchtrup \& Torrilhon (2003) we obtained a regularization of the Grad equations by means of a Chapman-Enskog-like expansion around a pseudo-equilibrium (Karlin et al. 1998; Karlin \& Gorban 2002) given by prescribed values of the 13 variables $\rho_{A}$ to obtain

$$
\begin{aligned}
m_{i j k}= & -2 \frac{\mu}{p}\left[R T \frac{\partial \sigma_{\langle i j}}{\partial x_{k\rangle}}-R T \sigma_{\langle i j} \frac{\partial \ln \varrho}{\partial x_{k\rangle}}+\frac{4}{5} q_{\langle i} \frac{\partial v_{j}}{\partial x_{k\rangle}}-\frac{\sigma_{\langle i j}}{\varrho} \frac{\partial \sigma_{k\rangle l}}{\partial x_{l}}\right], \\
R_{i j}= & -\frac{24}{5} \frac{\mu}{p}\left[R T \frac{\partial q_{\langle i}}{\partial x_{j\rangle}}+R q_{\langle i} \frac{\partial T}{\partial x_{j\rangle}}-R T q_{\langle i} \frac{\partial \ln \varrho}{\partial x_{j\rangle}}-\frac{1}{\rho} q_{\langle i} \frac{\partial \sigma_{j\rangle k}}{\partial x_{k}}\right. \\
& \left.+\frac{5}{7} R T\left(\sigma_{k\langle i} \frac{\partial v_{j\rangle}}{\partial x_{k}}+\sigma_{k\langle i} \frac{\partial v_{k}}{\partial x_{j\rangle}}-\frac{2}{3} \sigma_{i j} \frac{\partial v_{k}}{\partial x_{k}}\right)-\frac{5}{6} \frac{\sigma_{i j}}{\varrho} \frac{\partial q_{k}}{\partial x_{k}}-\frac{5}{6} \frac{\sigma_{i j}}{\varrho} \sigma_{k l} \frac{\partial v_{k}}{\partial x_{l}}\right], \\
\Delta= & -12 \frac{\mu}{p}\left[R T \frac{\partial q_{k}}{\partial x_{k}}+\frac{5}{2} R q_{k} \frac{\partial T}{\partial x_{k}}-R T q_{k} \frac{\partial \ln \varrho}{\partial x_{k}}-\frac{1}{\varrho} q_{j} \frac{\partial \sigma_{j k}}{\partial x_{k}}+R T \sigma_{i j} \frac{\partial v_{i}}{\partial x_{j}}\right] .
\end{aligned}
$$

In the resulting system (2.1)-(2.3) with (2.5), second-order derivatives are introduced in the balance equations of stress tensor and heat flux which lead to a regularization of the original 13-moment case of Grad. The new terms are of super-Burnett order, and the system contains the Navier-Stokes-Fourier, Burnett and super-Burnett equations in a stable way. These features are discussed in the following sections, and we shall also compare the R13 equations with results due to other authors, in particular with the augmented Burnett equations by Zhong et al. (1991, 1993), and the regularized Burnett equations of Jin \& Slemrod (2001), Jin et al. (2002).

The R13 equations were derived from the Boltzmann equations for the special case of Maxwell molecules, that is particles that interact in a repulsive fifth-power potential. The corresponding viscosity is proportional to temperature:

$$
\mu=\mu_{0}\left(\frac{T}{T_{0}}\right)^{s}
$$

with $s=1$. It is well known (Bird 1998) that the viscosity is also of this form for other interaction potentials with just an adjustment of the exponent $s$. In particular $s=1 / 2$ is computed for hard spheres, and $s \approx 0.8$ is measured for argon. For the computation of the shock structure in the second half of the paper, we shall use (2.6) with $s$ as a variable parameter to adjust the R13 equations to other interaction potentials. For the discussion of the Chapman-Enskog expansions below we shall use only $s=1$.

\subsection{Chapman-Enskog expansions}

In Struchtrup \& Torrilhon (2003) we showed that a Chapman-Enskog expansion of the linearized R13 equations yields the linearized Navier-Stokes-Fourier, Burnett, and super-Burnett equations. Here, we shall extend this to the nonlinear one-dimensional case.

The idea of the Chapman-Enskog expansion is to expand the distribution function in a series in a formal smallness parameter $\varepsilon$, which represents the Knudsen number when one considers dimensionless quantities, as

$$
f=f^{(0)}+\varepsilon f^{(1)}+\varepsilon^{2} f^{(2)}+\varepsilon^{3} f^{(3)}+\cdots
$$

where the $f^{(\alpha)}$ are obtained from the Boltzmann equation (Reinecke \& Kremer 1990; Chapman \& Cowling 1970). In our case, we operate at the level of moments and 
moment equations, and thus we expand pressure deviator and heat flux in series as

$$
\begin{gathered}
\sigma_{i j}=\sigma_{i j}^{(0)}+\varepsilon \sigma_{i j}^{(1)}+\varepsilon^{2} \sigma_{i j}^{(2)}+\varepsilon^{3} \sigma_{i j}^{(3)}+\cdots, \\
q_{i}=q_{i}^{(0)}+\varepsilon q_{i}^{(1)}+\varepsilon^{2} q_{i}^{(2)}+\varepsilon^{3} q_{i}^{(3)}+\cdots .
\end{gathered}
$$

The above expressions are inserted into the balance equations (2.2) and (2.3) and terms with equal powers in $\varepsilon$ are equated to find the $\sigma_{i j}^{(\alpha)}, q_{i}^{(\alpha)}$. Note that the proper scaling requires replacing $\mu$ by $\varepsilon \mu$ in (2.2), (2.3), (2.5). The formal parameter $\varepsilon$ will be set to unity at the end of the calculations.

In the Chapman-Enskog method it is customary to express the time derivatives of $\sigma_{i j}^{(\alpha)}, q_{i}^{(\alpha)}$ by time derivatives of the hydrodynamic variables $\varrho, T, v_{i}$. Some details on how this is done successively for the linear case can be found in Struchtrup \& Torrilhon (2003).

From the R13 equations as given above with $s=1$ (Maxwell molecules) we find the Euler equations at zeroth order,

$$
\sigma_{i j}^{(0)}=q_{i}^{(0)}=0,
$$

and the first-order corrections are the Navier-Stokes-Fourier equations

$$
\sigma_{i j}^{(1)}=-2 \mu \frac{\partial v_{\langle i}}{\partial x_{j\rangle}} \quad \text { and } \quad q_{i}^{(1)}=-\frac{15}{4} R \mu \frac{\partial T}{\partial x_{i}} .
$$

The second-order terms yield the Burnett equations for Maxwell molecules, that can be written as

$$
\begin{aligned}
\sigma_{i j}^{(2)}= & \frac{\mu^{2}}{p}\left[R \frac{\partial^{2} T}{\partial x_{\langle i} \partial x_{j\rangle}}-2 \frac{R T}{\varrho} \frac{\partial^{2} \varrho}{\partial x_{\langle i} \partial x_{j\rangle}}+2 \frac{R T}{\varrho^{2}} \frac{\partial \varrho}{\partial x_{\langle i}} \frac{\partial \varrho}{\partial x_{j\rangle}}-2 \frac{R}{\varrho} \frac{\partial T}{\partial x_{\langle i}} \frac{\partial \varrho}{\partial x_{j\rangle}}\right. \\
& \left.+3 \frac{R}{T} \frac{\partial T}{\partial x_{\langle i}} \frac{\partial T}{\partial x_{j\rangle}}+\frac{10}{3} S_{i j} \frac{\partial v_{k}}{\partial x_{k}}-4 S_{k\langle i} \frac{\partial v_{k}}{\partial x_{j\rangle}}-2 \frac{\partial v_{k}}{\partial x_{\langle i}} \frac{\partial v_{j\rangle}}{\partial x_{k}}+8 S_{k\langle i} S_{j\rangle k}\right]
\end{aligned}
$$

for the stress tensor, and

$$
\begin{aligned}
q_{i}^{(2)}= & \frac{\mu^{2}}{p}\left[-\frac{13}{4} R T \frac{\partial^{2} v_{k}}{\partial x_{i} \partial x_{k}}+\frac{3}{2} R T \frac{\partial^{2} v_{i}}{\partial x_{k} \partial x_{k}}-3 \frac{R T}{\varrho} S_{i j} \frac{\partial \varrho}{\partial x_{j}}\right. \\
& \left.-\frac{25}{8} R \frac{\partial v_{k}}{\partial x_{k}} \frac{\partial T}{\partial x_{i}}+\frac{15}{8} R \frac{\partial v_{k}}{\partial x_{i}} \frac{\partial T}{\partial x_{k}}+\frac{105}{8} R \frac{\partial v_{i}}{\partial x_{k}} \frac{\partial T}{\partial x_{k}}\right]
\end{aligned}
$$

for the heat flux, where we have used the abbreviation

$$
S_{i j}=\frac{\partial v_{\langle i}}{\partial x_{j\rangle}} .
$$

It is not surprising that the Burnett equations arise from the second-order expansion of the R13 equations, since it is an established fact that the Burnett equations can be obtained from Grad's 13-moment equations (Reinecke \& Kremer 1990, 1996; Struchtrup 2004a), i.e. with the Grad closure (2.4).

A closer inspection of the closure relations (2.5) of the R13 equations shows that these contribute only terms of super-Burnett order. The derivation of the superBurnett equations is a very cumbersome task, and they are quite difficult to find in the literature. Thus, we expanded the R13 equations for two special cases only: the three-dimensional linear equations and the one-dimensional nonlinear equations. For the three-dimensional linear case details on the computation can be found in 
Struchtrup \& Torrilhon (2003). For reference we repeat the equations here:

$$
\begin{aligned}
\sigma_{i j}^{(3)} & =\frac{\mu^{3}}{p^{2}}\left(\frac{5}{3} R T \frac{\partial^{2}}{\partial x_{\langle i} \partial x_{j\rangle}} \frac{\partial v_{k}}{\partial x_{k}}-\frac{4}{3} R T \frac{\partial^{2}}{\partial x_{k} \partial x_{k}} \frac{\partial v_{\langle i}}{\partial x_{j\rangle}}\right), \\
q_{i}^{(3)} & =\frac{\mu^{3}}{p^{2}}\left(-\frac{157}{16} R T \frac{\partial^{3} \theta}{\partial x_{i} \partial x_{k} \partial x_{k}}-\frac{5}{8} \frac{R^{2} T^{2}}{\rho} \frac{\partial^{3} \varrho}{\partial x_{i} \partial x_{k} \partial x_{k}}\right) .
\end{aligned}
$$

These are the same as the equations that Shavaliyev (1993) found directly from the Boltzmann equation for Maxwell molecules.

The nonlinear and one-dimensional super-Burnett equations as derived from the R13 equations are, for the stress tensor,

$$
\begin{aligned}
\sigma_{\langle 11\rangle}^{(3)}= & \frac{\mu^{3}}{p^{2}}\left[\frac{47}{3} \frac{R}{\rho} \frac{\partial \rho}{\partial x} \frac{\partial T}{\partial x} \frac{\partial v}{\partial x}-\frac{64}{9} \frac{R T}{\rho^{2}}\left(\frac{\partial \rho}{\partial x}\right)^{2} \frac{\partial v}{\partial x}+\frac{40}{9} \frac{R T}{\rho} \frac{\partial^{2} \rho}{\partial x^{2}} \frac{\partial v}{\partial x}-\frac{2}{3} \frac{R T}{\rho} \frac{\partial \rho}{\partial x} \frac{\partial^{2} v}{\partial x^{2}}\right. \\
& \left.-7 \frac{R}{T}\left(\frac{\partial T}{\partial x}\right)^{2} \frac{\partial v}{\partial x}-\frac{47}{9} R \frac{\partial T}{\partial x} \frac{\partial^{2} v}{\partial x^{2}}-\frac{31}{9} R \frac{\partial^{2} T}{\partial x^{2}} \frac{\partial v}{\partial x}+\frac{2}{9} R T \frac{\partial^{3} v}{\partial x^{3}}+\frac{16}{27}\left(\frac{\partial v}{\partial x}\right)^{3}\right],
\end{aligned}
$$

and for the heat flux

$$
\begin{aligned}
q_{1}^{(3)}= & \frac{\mu^{3}}{p^{2}}\left[-\frac{2913}{112} R \frac{\partial T}{\partial x}\left(\frac{\partial v}{\partial x}\right)^{2}+\frac{188}{21} \frac{R T}{\rho} \frac{\partial \rho}{\partial x}\left(\frac{\partial v}{\partial x}\right)^{2}+\frac{199}{56} R T \frac{\partial^{2} v}{\partial x^{2}} \frac{\partial v}{\partial x}\right. \\
& +\frac{917}{8} \frac{R^{2}}{\rho} \frac{\partial \rho}{\partial x}\left(\frac{\partial T}{\partial x}\right)^{2}-\frac{1137}{16} \frac{R^{2} T}{\rho^{2}}\left(\frac{\partial \rho}{\partial x}\right)^{2} \frac{\partial T}{\partial x}+\frac{397}{16} \frac{R^{2} T}{\rho} \frac{\partial \rho}{\partial x} \frac{\partial^{2} T}{\partial x^{2}} \\
& +\frac{701}{16} \frac{R^{2} T}{\rho} \frac{\partial^{2} \rho}{\partial x^{2}} \frac{\partial T}{\partial x}-\frac{813}{16} \frac{R^{2}}{T}\left(\frac{\partial T}{\partial x}\right)^{3}-\frac{1451}{16} R^{2} \frac{\partial T}{\partial x} \frac{\partial^{2} T}{\partial x} \\
& \left.-\frac{157}{16} R^{2} T \frac{\partial^{3} T}{\partial x^{3}}-\frac{41}{8} \frac{R^{2} T^{2}}{\rho^{2}} \frac{\partial^{2} \rho}{\partial x^{2}} \frac{\partial \rho}{\partial x}-\frac{5}{8} \frac{R^{2} T^{2}}{\rho} \frac{\partial^{3} \rho}{\partial x^{3}}+\frac{23}{4} \frac{R^{2} T^{2}}{\rho^{3}}\left(\frac{\partial \rho}{\partial x}\right)^{3}\right]
\end{aligned}
$$

Those terms in (2.12) and (2.13) that are not underlined agree with those given by Shavaliyev (1993), and Fiscko \& Chapman (1989), while there are slight differences in the magnitude of the coefficients of the underlined terms. In equation (2.12) for the stress, all coefficients agree with those of Fiscko \& Chapman, while Shavaliyev has in the underlined terms

$$
\left[-\frac{40}{3}, \frac{32}{3}\right] \text { instead of }\left[-\frac{64}{9}, \frac{40}{9}\right] .
$$

In the equation for the heat flux (2.13), Shavaliyev gives the coefficients in the three underlined terms as

$$
\left[-\frac{9005}{168}, \frac{271}{21}, \frac{421}{42}\right] \quad \text { instead of }\left[-\frac{2913}{112}, \frac{188}{21}, \frac{199}{56}\right]
$$

while Fiscko \& Chapman have

$$
\left[-\frac{8035}{336}, \frac{166}{21}, \frac{949}{168}\right]
$$

Note that the signs of the coefficients agree in all three cases. 
While we cannot judge the results of Shavaliyev and Fiscko \& Chapman without redoing their computations, we state that it is not unlikely that small algebraic errors occurred in their derivations, which are based on the Boltzmann equation. We checked the R13 equations carefully, and used the computer algebra system Mathematica $\left.{ }^{(}\right)$ in order to expand the one-dimensional R13 equations into Chapman-Enskog series. The computation of the super-Burnett relations from the R13 equations is much simpler than from the Boltzmann equations.

As a summary of this section we believe it is safe to say that the R13 equations agree up to the super-Burnett order with the Boltzmann equation. Note that the classical 13-moment equations of Grad do not agree up to super-Burnett order.

Moreover, the R13 equations have several advantages over the Burnett and superBurnett equations: (i) They can be derived much more easily, and faster, so that errors can be excluded with higher certainty. (ii) The R13 equations contain only space derivatives of first and second-order while the super-Burnett equations contain derivatives of up to fourth order. Thus, the R13 equations fit existing numerical methods more conveniently. Note that their mathematical structure is very similar to the NSF equations, so that methods for these can also be used for solving the R13 equations. (iii) Most important, however, is the fact that the R13 equations are linearly stable as was shown in Struchtrup \& Torrilhon (2003), while the Burnett and super-Burnett equations are linearly unstable (Bobylev 1982).

The question of why the R13 equations are stable, while Burnett and super-Burnett equations are unstable, is not easy to answer, since there is no clear reason why the Chapman-Enskog expansion leads to unstable equations in the first place. It seems that a first-order Chapman-Enskog expansion leads generally to stable equations, while higher-order expansions generally yield unstable equations. However, some exceptions are known.

The Burnett equations for the BGK model, a simplification of the Boltzmann collision term (Bhatnagar, Gross \& Krook 1954), turn out to be stable (Agarwal, Yun \& Balakrishnan 2001), but are limited in their usefulness due to the wrong Prandtl number in the BGK model, where $\operatorname{Pr}=1$. The more general Ellipsoidal Statistical (ES)-BGK model (Holway 1996) allows one to adjust the Prandtl number, and the stability of the corresponding ES-BGK-Burnett equations depends on the value of the Prandtl number, see Zheng \& Struchtrup (2003). Prandtl numbers $1 \leqslant P r \leqslant 5 / 4$ and $\operatorname{Pr} \rightarrow \infty$ yield stable Burnett equations, while all other values of $P r$, including the value measured for gases, $P r=2 / 3$, yield unstable Burnett equations. Karlin \& Gorban (2002) showed that the Chapman-Enskog expansion of Grad's moment equations yields stable results, when an infinite number of terms is considered, while truncated expansions are unstable.

Relative to Chapman-Enskog expansions, the R13 equations result from a firstorder expansion (around a non-equilibrium state), just like the Navier-Stokes equations (around the equilibrium), and that might be one reason for their stability. Note also, that the R13 equations contain terms of higher order in $K n$, indeed an infinite number of these, as follows from accounting for higher terms in the ChapmanEnskog expansion. One might say that these higher-order terms stabilize the equations.

\subsection{Augmented Burnett equations of Zhong et al.}

The instability of the original Burnett equations lead Zhong et al. to a modification whereby they added some terms of super-Burnett order to the Burnett equations with the goal of stabilizing the equations, and they termed their new set of equations "augmented Burnett equations" (Zhong et al. 1991, 1993). For the one-dimensional 
case they added the following linear terms to the Burnett equations:

$$
\left.\begin{array}{rl}
\sigma_{\langle 11\rangle}^{(A)} & =\frac{\mu^{3}}{p^{2}}\left(\frac{2}{9} R T \frac{\partial^{3} v}{\partial x^{3}}\right), \\
q_{1}^{(A)} & =\frac{\mu^{3}}{p^{2}}\left(\frac{11}{16} R^{2} T \frac{\partial^{3} T}{\partial x^{3}}-\frac{5}{8} \frac{R^{2} T^{2}}{\rho} \frac{\partial^{3} \rho}{\partial x^{3}}\right) .
\end{array}\right\}
$$

Comparison with the true super-Burnett terms (2.11) shows that Zhong et al. have in the heat flux a coefficient of $11 / 16$ instead of $-157 / 16$, citing Wang-Chang (1970) as a source of the coefficient. This coefficient contradicts all other results on the super-Burnett equations as cited in the previous section, and must be considered as not being based on the Chapman-Enskog expansion of the Boltzmann equation. The important difference to the proper super-Burnett terms is the difference in sign, which yields stability in time.

Furthermore, Zhong et al. generalize their augmented equations in an ad hoc manner to three dimensions as

$$
\left.\begin{array}{rl}
\sigma_{i j}^{(A)} & =\frac{\mu^{3}}{p^{2}}\left(\frac{1}{3} R T \frac{\partial^{2}}{\partial x_{k} \partial x_{k}} \frac{\partial v_{\langle i}}{\partial x_{j\rangle}}\right), \\
q_{i}^{(A)} & =\frac{\mu^{3}}{p^{2}}\left(\frac{11}{16} R^{2} T \frac{\partial^{3} T}{\partial x_{i} \partial x_{k} \partial x_{k}}-\frac{5}{8} \frac{R^{2} T^{2}}{\varrho} \frac{\partial^{3} \rho}{\partial x_{i} \partial x_{k} \partial x_{k}}\right) .
\end{array}\right\}
$$

Comparison with (2.11) shows that there is not only the wrong factor $11 / 16$ instead of $-157 / 16$ in the heat flux, but also that the three-dimensional ad hoc term $\sigma_{i j}^{(a)}$ does not have the proper tensorial structure, since it differs from the super-Burnett relation $(2.11 a)$.

In the following we shall show that the augmented Burnett equations are stable only in time but not in space. Therefore, we review some of the basics of the test of linear stability. In the one-dimensional case, the variables of the augmented Burnett equations are mass density, temperature and velocity, $\phi=\{\varrho, T, v\}$. For these, one assumes plane wave solutions of the form

$$
\phi=\tilde{\phi} \exp \{i(\omega t-k x)\}
$$

where $\tilde{\phi}$ is the complex amplitude of the wave, $\omega$ is its frequency, and $k$ is its wavenumber. The equations can be written as

$$
\mathscr{A}(\omega, k) \tilde{\phi}=0
$$

and non-trivial solutions require

$$
\operatorname{det}[\mathscr{A}(\omega, k)]=0 .
$$

The resulting relation between $\omega$ and $k$ is the dispersion relation.

If a disturbance in space is considered, the wavenumber $k$ is real, and the frequency is complex, $\omega=\omega_{r}(k)+\mathrm{i} \omega_{i}(k)$. The phase velocity $v_{p h}$ and damping $\alpha$ of the corresponding waves are given by

$$
v_{p h}=\frac{\omega_{r}(k)}{k} \text { and } \alpha=\omega_{i}(k) .
$$

Stability requires damping, and thus $\omega_{i}(k) \geqslant 0$. If, however, $\omega_{i}(k)<0$ then a small disturbance in space will blow up in time. 

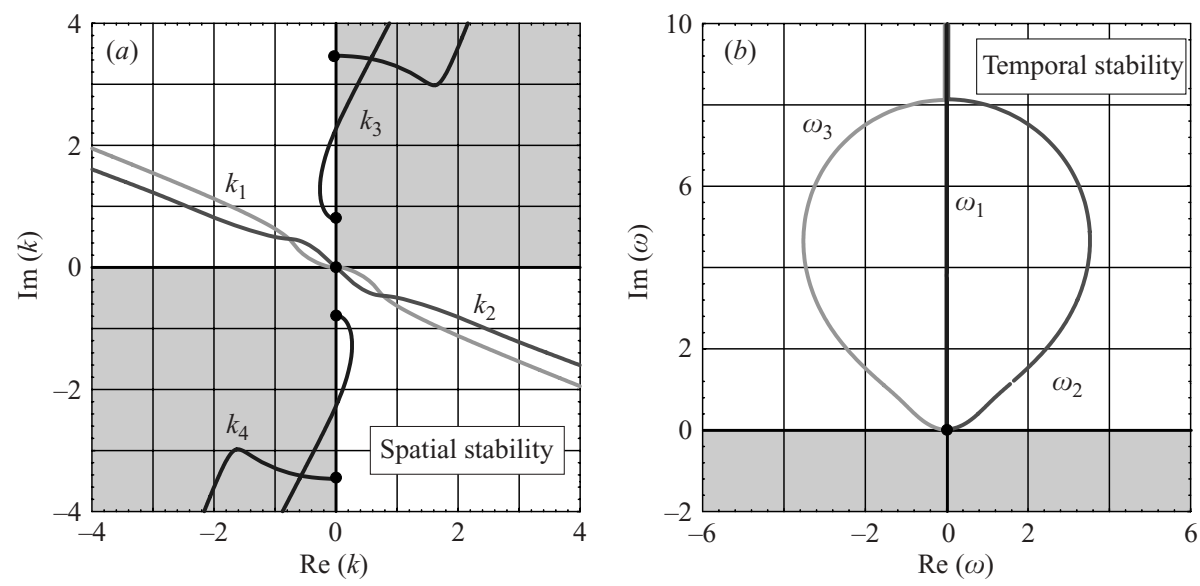

FIGURE 1. Stability of the augmented Burnett equations: $(a) k(\omega)$ in the complex plane with $\omega$ as curve parameter, and $(b) \omega(k)$ in the complex plane with $k$ as parameter. Curves running through the grey-shaded areas correspond to unstable processes.

If a disturbance in time at a given location is considered, the frequency $\omega$ is real, while the wavenumber is complex, $k=k_{r}(\omega)+\mathrm{i} k_{i}(\omega)$. The phase velocity $v_{p h}$ and damping $\alpha$ of the corresponding waves are given by

$$
v_{p h}=\frac{\omega}{k_{r}(\omega)} \text { and } \alpha=-k_{i}(\omega)
$$

For a wave travelling in the positive $x$-direction $\left(k_{r}>0\right)$, the damping must be negative $\left(k_{i}<0\right)$, while for a wave travelling in the negative $x$-direction $\left(k_{r}<0\right)$, the damping must be positive $\left(k_{i}>0\right)$. If this condition is not fulfilled, then a disturbance in time at some point will lead to a much larger disturbance at other locations.

Thus, in order to test the stability of a given set of equations, one has to perform two tests, for stability in time and stability in space. $\dagger$ However, for the Burnett and super-Burnett equations, most authors only consider the stability in time (Bobylev 1982; Zhong et al. 1991), and ignore the stability in space.

In Struchtrup \& Torrilhon (2003) we performed both tests for the R13, Burnett, and super-Burnett equations, and showed that the latter two are unstable for both, while the R13 equations are stable for both. The different sign in (2.14) yields stability in time, as stated by Zhong et al., but does not remove the spatial stability of the Burnett equations. This becomes evident in figure 1, which shows the two solutions $k(\omega)$ and $\omega(k)$ in the complex plane. Instability occurs when one branch of the solution enters the grey-shaded areas. This does not happen for $\omega(k)$, so that temporal stability is ensured, but the branches $k_{3}(\omega)$ and $k_{4}(\omega)$ pass through the grey region, and thus spatial stability is violated.

The conclusion of this section is that the augmented Burnett equations cannot be justified on the grounds of the Boltzmann equation, and must be considered as an ad hoc correction, which fulfils its goal only partly, since the equations are temporally stable, but spatially unstable.

$\dagger$ An easy example for an equation which is stable in space but unstable in time is $\partial y / \partial t-\partial^{2} y / \partial x^{2}+\partial^{3} y / \partial t \partial x^{2}=0$. 
The R13 equations are superior on two counts: (i) they are stable with respect to both tests, and (ii) they are accurate to super-Burnett order, while the augmented Burnett equations are accurate only to Burnett order.

\subsection{Regularized Burnett equations of Jin \& Slemrod}

Recently, Jin \& Slemrod introduced a regularization method for the Burnett equations, which is based on Grad's 13-moment method and the requirement of a positive entropy generation, see Jin \& Slemrod (2001), Jin et al. (2002). Their method provides a set of stable equations, which agree with Chapman-Enskog expansions of the Boltzmann equation up to Burnett order, but not to super-Burnett order. Described briefly, their equations are Grad's 13-moment equations plus additional terms of super-Burnett order, and thus there is a strong resemblance to the R13 equations. In order to point out the main differences, we compare the regularized Burnett equations with the R13 equations in linearized and dimensionless form (see Struchtrup \& Torrilhon (2003) for definitions of the dimensionless quantities). In both cases, the relevant equations are the conservation laws for mass, momentum and energy plus the balance laws for the stress deviator and heat flux, which we write as

$$
\begin{gathered}
\frac{\partial \sigma_{i j}}{\partial t}+\frac{4}{5} \frac{\partial q_{\langle i}}{\partial x_{j\rangle}}+2 \frac{\partial v_{\langle i}}{\partial \hat{x}_{j\rangle}}+\Pi_{\langle i j\rangle}=-\frac{\sigma_{i j}}{\mathrm{Kn}}, \\
\frac{\partial q_{i}}{\partial t}+\frac{5}{2} \frac{\partial T}{\partial x_{i}}+\frac{\partial \sigma_{i k}}{\partial x_{k}}+\Gamma_{i}=-\frac{2}{3} \frac{q_{i}}{\mathrm{Kn}} .
\end{gathered}
$$

The derivation of the balance equations for $\sigma_{i j}$ and $q_{i}$ as moments of the Boltzmann equation requires that $\Pi_{\langle i j\rangle}$ and $\Gamma_{i}$ are of the form

$$
\Pi_{\langle i j\rangle}=\frac{\partial m_{\langle i j k\rangle}}{\partial x_{k}}, \quad \Gamma_{i}=\frac{1}{2} \frac{\partial R_{\langle i k\rangle}}{\partial x_{k}}+\frac{1}{6} \frac{\partial \Delta}{\partial x_{i}} .
$$

The R13 equations indeed are of this form, with explicit functions for the constitutive quantities $m_{\langle i j k\rangle}, R_{\langle i j\rangle}$ and $\Delta$. When we use equations (2.5) in their non-dimensional linearized form we obtain

$$
\left.\begin{array}{l}
\Pi_{\langle i j\rangle}^{(R 13)}=-\frac{2}{3} \mathrm{Kn} \frac{\partial^{2} \sigma_{i j}}{\partial x_{k} \partial x_{k}}-\frac{4}{5} \mathrm{Kn} \frac{\partial^{2} \sigma_{k\langle i}}{\partial x_{j\rangle} \partial x_{k}}, \\
\Gamma_{i}^{(R 13)}=-\frac{6}{5} \mathrm{Kn} \frac{\partial^{2} q_{i}}{\partial x_{k} \partial x_{k}}-\frac{12}{5} \mathrm{Kn} \frac{\partial^{2} q_{k}}{\partial x_{k} \partial x_{i}} .
\end{array}\right\}
$$

Jin \& Slemrod obtain

$$
\left.\begin{array}{rl}
\Pi_{\langle i j\rangle}^{(J / S)} & =\frac{\bar{\omega}_{4}}{5} \operatorname{Kn} \frac{\partial^{2} \sigma_{i j}}{\partial x_{k} \partial x_{k}}, \\
\Gamma_{i}^{(J / S)} & =\frac{8 \bar{\theta}_{4}}{45} \operatorname{Kn} \frac{\partial^{2} q_{i}}{\partial x_{k} \partial x_{k}},
\end{array}\right\}
$$

where $\bar{\omega}_{4}$ and $\bar{\theta}_{4}$ are unknown coefficients (real numbers) that can be obtained from fitting to experiments. Clearly, their equations are not of the required form (2.18), and this is one of the reasons why the addition of terms of super-Burnett order does not lead to the recovery of the super-Burnett equations. Note that a comparison between the one-dimensional equations of both theories allows one to identify the unknown coefficients of Jin \& Slemrod as

$$
\bar{\omega}_{4}=6, \quad \bar{\theta}_{4}=\frac{81}{4} .
$$


The advantages of the R13 equations over the regularized Burnett equations are: (i) The R13 equations are accurate to super-Burnett order while the regularized Burnett-equations are accurate only to Burnett order. (ii) No unknown coefficients appear in the R13 equations. However, the regularized Burnett equations can be related to an entropy with positive production, while, so far, an appropriate entropy for the R13 equations has not been found.

In the following the shock structure profiles of the regularized Burnett equations are not considered, since our numerical method is not directly applicable to the equations in Jin \& Slemrod (2001). Furthermore, the results of the regularized Burnett equations are subject to fitting parameters in general, which is not the case for the other Burnett models. Shock profiles obtained with the regularized Burnett equations may be found in Jin et al. (2002).

\section{Shock structure computations}

\subsection{Stationary one-dimensional equations}

The shock profile connects the equilibrium states of density $\rho_{0}$, velocity $v_{0}$, and temperature $T_{0}$ before the shock at $x \rightarrow-\infty$ with the equilibrium $\rho_{1}, v_{1}, T_{1}$ behind the shock at $x \rightarrow \infty$. The process is modelled as one-dimensional flow. Hence, velocity, pressure deviator and heat flux have only a single non-trivial component in the direction normal to the shock wave. The field quantities are related to their values at $x \rightarrow-\infty$ by the definition of the non-dimensional quantities

$$
\hat{\rho}=\frac{\rho}{\rho_{0}}, \quad \hat{v}=\frac{v}{\sqrt{R T_{0}}}, \quad \hat{T}=\frac{T}{T_{0}}, \quad \hat{\sigma}=\frac{\sigma}{\rho_{0} R T_{0}}, \quad \hat{q}=\frac{q}{\rho_{0}{\sqrt{R T_{0}}}^{3}} .
$$

Here, $\hat{\sigma}=\sigma_{\langle 11\rangle}$ represents the non-trivial component of the pressure deviator, called stress in the following, and $\hat{q}$ denotes the normal heat flux.

A dimensionless space variable is introduced as

$$
\hat{x}=\frac{x \rho_{0} \sqrt{R T_{0}}}{\mu_{0}}
$$

where $\mu_{0}$ is the viscosity of the state before the shock. From the viscosity follows the mean free path, see e.g. Bird (1998) or Chapman \& Cowling (1970), calculated for $x \rightarrow-\infty$, namely

$$
\bar{\lambda}_{0}=\frac{4}{5} \frac{\mu_{0}}{\rho_{0} \sqrt{\frac{1}{8} \pi R T_{0}}}
$$

Thus, the relation

$$
\frac{x}{\bar{\lambda}_{0}}=\frac{5}{4} \sqrt{\frac{1}{8} \pi} \hat{x} \approx 0.783 \hat{x}
$$

holds for our dimensionless space variable. In the plots below we will always use $x / \lambda_{0}$ as space variable. For the sake of simplicity we drop the hats from non-dimensional variables in what follows.

The profile can be considered in the frame of reference moving with the shock and is described by stationary field equations. Thus, in the one-dimensional formulation, the equations of the regularized 13-moment case for monatomic gases (2.1)-(2.3) can 
be written as

$$
\begin{aligned}
\frac{\mathrm{d}}{\mathrm{d} x}(\rho v) & =0 \\
\frac{\mathrm{d}}{\mathrm{d} x}\left(\rho v^{2}+\rho T+\sigma\right) & =0 \\
\frac{\mathrm{d}}{\mathrm{d} x}\left(\rho v^{3}+5 \rho T v+2 v \sigma+2 q\right) & =0 \\
\frac{\mathrm{d}}{\mathrm{d} x}\left(\rho v^{3}+3 \rho T v+3 \sigma v+\frac{6}{5} q+m\right) & =-\rho \alpha(T) \sigma \\
\frac{\mathrm{d}}{\mathrm{d} x}\left(\frac{1}{2} \rho v^{4}+4 \rho T v^{2}+\frac{5}{2} \sigma v^{2}+\frac{16}{5} q v+\frac{7}{2} T \sigma+\frac{5}{2} \rho T^{2}+m v+B\right) & =-\rho \alpha(T)\left(\sigma v+\frac{2}{3} q\right) .
\end{aligned}
$$

These equations have production proportional to $\alpha(T)=R T / \mu(T)$ determined by the particle interaction potential. Originally, the equations were derived for Maxwell molecules where $\alpha$ is a constant. As mentioned in $\S 2$ we extent the equations to more realistic cases by assuming that $\alpha$ depends on the temperature. In the present dimensionless form $\alpha(T)$ may be written as

$$
\alpha(T)=T^{(1-s)},
$$

where $s$ is the viscosity exponent of the gas under consideration.

The quantities $m=m_{\langle 111\rangle}$ and $B=\frac{1}{2} R_{\langle 11\rangle}+\frac{1}{6} \Delta$, introduced in (3.5) follow from the one-dimensional forms of the constitutive equations (2.5) and are

$$
\begin{aligned}
& m=-\frac{6}{5} \frac{\delta}{\rho \alpha(T)}\left[\left(T-\frac{\sigma}{\rho}\right) \frac{\mathrm{d} \sigma}{\mathrm{d} x}-\sigma T \frac{\mathrm{d} \ln \rho}{\mathrm{d} x}+\frac{8}{15} q \frac{\mathrm{d} v}{\mathrm{~d} x}\right] \\
& B=-\frac{18}{5} \frac{\delta}{\rho \alpha(T)}\left[\left(T-\frac{5}{9} \frac{\sigma}{\rho}\right) \frac{\mathrm{d} q}{\mathrm{~d} x}-\frac{q}{\rho} \frac{\mathrm{d} \sigma}{\mathrm{d} x}+\frac{11}{6} q \frac{\mathrm{d} T}{\mathrm{~d} x}-q T \frac{\mathrm{d} \ln \rho}{\mathrm{d} x}-\frac{5}{9}\left(\frac{\sigma}{\rho}-\frac{11}{7} T \sigma\right) \frac{\mathrm{d} v}{\mathrm{~d} x}\right],
\end{aligned}
$$

where the function $\alpha(T)$ again appears. The artificial parameter $\delta$ controls the transition between the classical 13-moment equations of Grad, $\delta=0$, and the regularized equations, $\delta=1$. The impact of this parameter is discussed in $\S 4.1$ below.

\subsection{Numerical method}

For the solution of (3.5) we follow a numerical strategy given in Weiss (1995). First of all, the number of variables of the system (3.5) will be reduced. In order to do so, the first three equations are integrated, starting before the shock, to give relations for density, stress and heat flux. The dimensionless variables in front of the shock are given by $\rho_{0}=1, v_{0}=\sqrt{5 / 3} M_{0}, T_{0}=1$ and the condition of equilibrium, $\sigma_{0}=q_{0}=0$. The Mach number of the shock

$$
M_{0}=\frac{v_{0}}{\sqrt{\frac{5}{3}}}
$$

acts as parameter. The density follows from the velocity by means of the mass balance as

$$
\rho(v)=\sqrt{\frac{5}{3}} \frac{M_{0}}{v},
$$


and the relations for stress $\sigma$ and heat flux $q$ as functions of velocity and temperature are

$$
\begin{aligned}
& \sigma(v, T)=1+\frac{5}{3} M_{0}^{2}-M_{0} \sqrt{\frac{5}{3}}\left(\frac{T}{v}+v\right), \\
& q(v, T)=\sqrt{\frac{5}{12}} M_{0}\left(\frac{5}{3} M_{0}^{2}+5 v^{2}-3 T\right)-v\left(1+\frac{5}{3} M_{0}^{2}\right) .
\end{aligned}
$$

With relations (3.10)-(3.12), equations $(3.5 d)$ and (3.5e) form two equations to determine velocity and temperature. After $m$ and $B$ in (3.5) are substituted by means of (3.7) and (3.8) the final system of equations may be written in the compact form

$$
\frac{\mathrm{d} F(v, T)}{\mathrm{d} x}=P(v, T)+\frac{\mathrm{d}}{\mathrm{d} x}\left(D(v, T) \frac{\mathrm{d} G(v, T)}{\mathrm{d} x}\right)
$$

where $F$ and $P$ are 2-vectors and the expression $D \mathrm{~d} G / \mathrm{d} x$ represent both equations (3.7) and (3.8). Shock structures for velocity and temperature are formally solutions of the system of ordinary differential equations (3.13) with the boundary conditions

$$
v_{0}=v(x \rightarrow-\infty)=\sqrt{\frac{5}{3}} M_{0}, \quad T_{0}=T(x \rightarrow-\infty)=1
$$

and

$$
v_{1}=v(x \rightarrow \infty)=\sqrt{\frac{5}{3}} \frac{M_{0}^{2}+3}{4 M_{0}}, \quad T_{1}=T(x \rightarrow \infty)=\frac{\left(5 M_{0}^{2}-1\right)\left(M_{0}^{2}+3\right)}{16 M_{0}^{2}} .
$$

The values behind the shock are given by the Rankine-Hugoniot relations. Since the right-hand side of (3.13) vanishes for both boundary conditions, shock structures represent, in terms of ordinary differential equations, orbits connecting stationary points in the phase space of (3.13). In Gilberg \& Paolucci (1953) shock structures of the NSF theory are calculated using detailed information about the orbit and the stationary points. Fortunately, it turned out that in our case the orbits are sufficiently well-behaved that we could use the straightforward finite difference method of Weiss (1995).

The shocks are assumed to relax fast, so that (3.14) and (3.15) may be prescribed at the boundaries of a finite computational domain. The computational domain is uniformly discretized into $N+2$ positions $x_{i}$ with $i=0,1,2 \ldots N+1$ and step size $\Delta x$, and the system (3.13) is evaluated at positions $x_{1}$ to $x_{N}$ with help of the discretization rules

$$
\begin{aligned}
\left.\frac{\mathrm{d} u}{\mathrm{~d} x}\right|_{i} & \rightarrow \frac{u_{i+1}-u_{i-1}}{2 \Delta x}, \\
\left.\frac{\mathrm{d}}{\mathrm{d} x}\left(a \frac{\mathrm{d} u}{\mathrm{~d} x}\right)\right|_{i} & \rightarrow \frac{\left(a_{i-1}+3 a_{i+1}\right) u_{i+1}-4\left(a_{i+1}+a_{i-1}\right) u_{i}+\left(3 a_{i-1}+a_{i+1}\right) u_{i-1}}{4 \Delta x^{2}} .
\end{aligned}
$$

The evaluations at position $x_{1}$ and $x_{N}$ require field values at $x_{0}$ and $x_{N+1}$ which are supplied by (3.14) and (3.15). Thus, the discretization of (3.13) yields $2 N$ coupled algebraic equations for the $N$ unknown values of velocity and temperature.

The resulting nonlinear system is solved by a quasi-Newtonian method described in Nowak \& Weimann (1990). As an initial guess for the values of velocity and temperature, discretizations of appropriate $\tanh (x)$ curves are used. Strictly speaking, the shock structure description as in (3.13) with (3.14) and (3.15) has no unique solution since the profile may be shifted arbitrarily along the space variable. In fact, 
the numerical method used shows convergence failures if the computational domain is chosen too large. For sufficiently small domains, however, converged solutions with relative accuracies in the magnitude of $10^{-9}$ could be achieved for the discretized system. Computational results for shock structures of the R13 equations presented in this paper have been obtained with $N=1000\left(\Delta x \approx 0.01 \lambda_{0}\right)$ on a standard PC Pentium-III within a few seconds.

The numerical method described above is also used to calculate the shock structures of the various Burnett-type equations. However, due to the instabilities of these equations the calculations must be conducted very carefully and are restricted to coarse discretizations with $\Delta x \approx \lambda_{0}$.

\section{Solution behaviour}

We next discuss the general behaviour of shock structure solutions of the R13 equations.

\subsection{Transition from Grad's 13-moment equations}

Grad's 13-moment case was derived as an improvement of the NSF theory in the description of rarefied flows. Unfortunately, the equations fail to describe continuous shock structures, since they suffer from a subshock in front of the shock beyond Mach number $M_{0}=1.65$, see $\operatorname{Grad}(1949,1958)$. This subshock grows with increasing Mach numbers and at $M_{0} \approx 3.5$ a second subshock appears in the middle of the shock. Both subshocks are artefacts of the hyperbolic nature of the 13-moment equations (Torrilhon 2000). It turned out that any hyperbolic moment theory will yield continuous shock structures only up to the Mach number corresponding to the highest characteristic velocity, see Ruggeri (1993) and Weiss (1995). Further validation of results with measurements shows that moment theories succeed in describing shock thickness data accurately only for Mach numbers far below this critical value. In particular, Grad's 13-moment case describes the shock thickness accurately only up to $M_{0} \approx 1.1$. Recent results from Au (2001) required up to 900 moments to calculate a smooth shock structure for $M_{0}=1.8$ that fits experimental data. For more information on shock structures in moment theories see Müller \& Ruggeri (1998).

One of the reasons to derive the new regularized 13-moment equations (R13) in Struchtrup \& Torrilhon (2003) was to obtain field equations which lead to smooth and stable shock structures for any Mach number. Since the equations are based on Grad's 13-moment case, it must be emphasized that physicality of the R13-solutions is still restricted to small Mach numbers. However, the range of validity is extended by including higher-order expansion terms in the R13 equations.

Figure 2 shows the transition to smooth shock structures for three different Mach numbers by means of the normalized velocity field $v_{N}$. The results are obtained with $s=1$, i.e. Maxwell molecules. The parameter $\delta$ in (3.7) and (3.8) is responsible for the transition. The structures with $\delta=0$ represent solutions of the classical 13moment case. At $M_{0}=1.651$ a kink at the beginning of the shock indicates that the highest characteristic velocity is reached before the shock. The kink develops into a pronounced subshock at $M_{0}=3$. In the case $M_{0}=6$ a second subshock is present towards the end of the structure.

The curves for $\delta=0.1$ mainly follow the results of Grad's 13-moment case. The subshocks are still clearly visible, albeit smoothed out by increased dissipation.

At $\delta=1$, however, the additional terms in the regularized 13-moment equations succeed in completely annihilating the subshocks and an overall smooth shock 


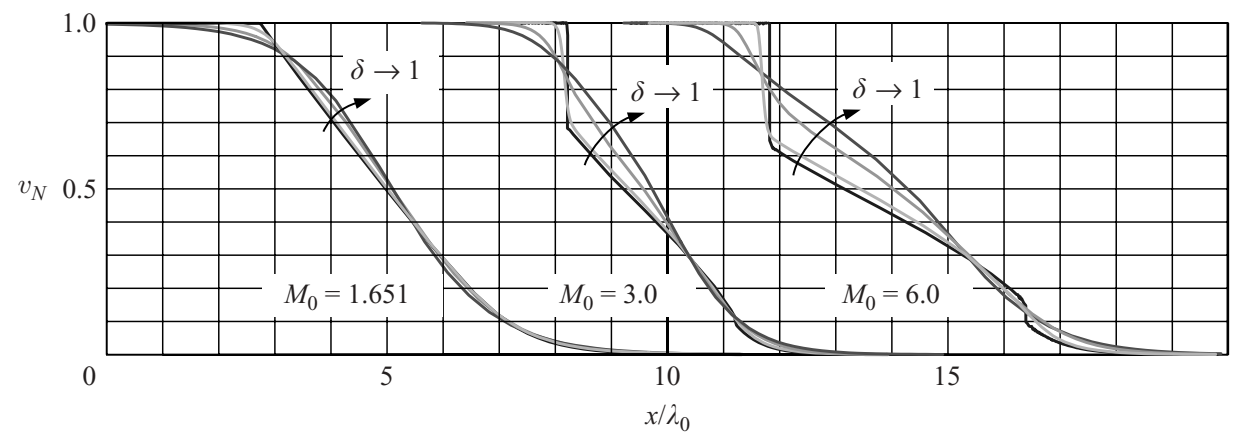

FIGURE 2. Regularization process of Grad's 13-moment equation. Profiles for three different Mach numbers are shown with different values of $\delta=0,0.1,0.5,1.0$. The results of Grad's equation $(\delta=0)$ exhibt kinks as well as up to two subshocks of increasing strength. These singularities vanish in the case of $\delta=1$.

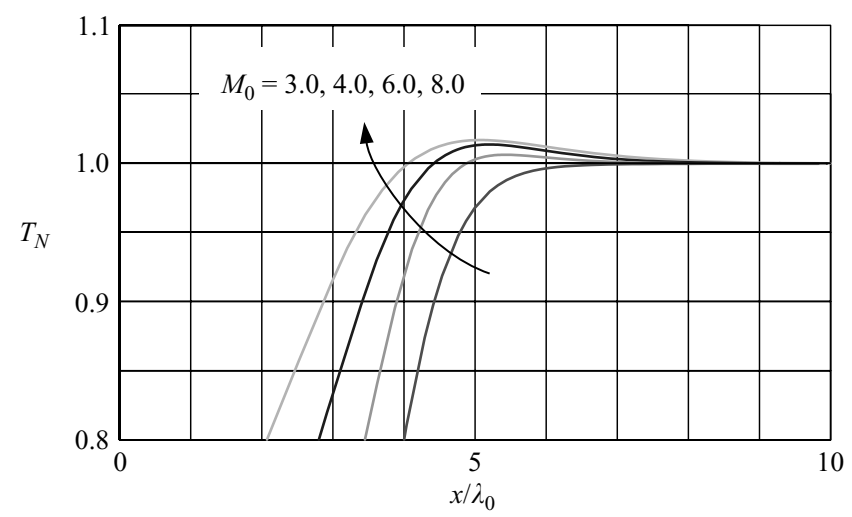

Figure 3. Magnification of the normalized temperature profile for several Mach numbers. The profile shows increasing temperature overshoots starting from Mach number $M_{0}=4$.

structure is obtained. At $M_{0}=6$ the R13 solution $(\delta=1)$ exhibits obvious asymmetries which start to appear in the structure for Mach numbers $M_{0}>3$, as discussed in $\S 5$. Since experiments as in Alsmeyer (1976) or DSMC simulations predict almost perfect S-shaped profiles we conclude that the validity of R13 solutions may be lost beyond Mach numbers $M_{0} \approx 3.0$.

\subsection{Temperature overshoot}

Figure 3 displays results of the temperature profile for Mach numbers up to $M_{0}=8$. The figure concentrates on the downstream part of the shock structure for a qualitative analysis. At Mach numbers $M_{0}>3$ the upstream part becomes more and more deformed which indicates the lack of physical validity of the equations in this area. The deformation can already be observed at the right-hand side of figure 2 and will be discussed in the next section.

All curves in figure 3 have been obtained as solutions of the complete R13 system, i.e. $\delta=1$, with a viscosity exponent $s=1$ and the temperature field has been normalized such that values $T_{0}$ and $T_{1}$ lie between 0 and 1 . As may be read from figure 3 the R13 equations qualitatively predict a temperature overshoot for high-Machnumber shocks. This phenomenon is known from experiments as well as from DSMC simulations, e.g. in Bird (1998), especially in the case of polyatomic gases due to the 
relaxation of the internal degrees of freedom. The effect is less pronounced but present in the case of monatomic gases. Here the overshoot is a result of a delayed relaxation of transversal (i.e.inside the shock plane) kinetic energy of the gas particles.

It is interesting to note that with the NSF theory no temperature overshoot for shock structures of monatomic gases can be obtained. This may be seen by considering the integrated equation (3.12) for $q$. Taylor expansion around the point $\left(v_{1}, T_{1}\right)$ yields

$$
\begin{aligned}
q(v, t) & \approx q\left(v_{1}, T_{1}\right)+\left.\frac{\partial q}{\partial v}\right|_{\left(v_{1}, T_{1}\right)}\left(v-v_{1}\right)+\left.\frac{\partial q}{\partial T}\right|_{\left(v_{1}, T_{1}\right)}\left(T-T_{1}\right) \\
& =-\frac{1}{4}\left(5 M_{0}^{2}-1\right)\left(v-v_{1}\right)-\sqrt{\frac{15}{4}} M_{0}\left(T-T_{1}\right) .
\end{aligned}
$$

The heat flux will be negative in the vicinity of the temperature overshoot where $T \rightarrow T_{1}+\Delta T$ and $v \rightarrow v_{1}+\Delta v$ with some small $\Delta T, \Delta v>0$. According to Fourier's law this yields a positive gradient of temperature in contradiction to the temperature overshoot where $\mathrm{d} T / \mathrm{d} x$ needs to be negative. Hence, a temperature overshoot is not possible in NSF theory. With R13, however, the temperature need not follow the heat flux, thus a temperature overshoot together with a negative heat flux is possible.

Similarly, the Burnett models are also capable of predicting a temperature overshoot since the heat flux is not related only to the temperature gradient. Indeed, the higherorder contributions (2.10) and (2.13) add cross-derivatives to the heat flux.

\section{Comparison with DSMC results}

In this section we shall compare the shock structures obtained with the regularized 13-moment equations to the results of the direct-simulation Monte-Carlo method (DSMC) of Bird (1998). For the DSMC results we used the shock structure code which is available from Bird's website. For the actual setup, such as interval length, upstream temperature, etc. we adopted the values of Pham-Van-Diep et al. (1991). Note that the calculation of a single low-Mach-number shock structure by a standard DSMC program takes several hours which is several orders of magnitude slower than the calculation by a continuum model.

We compare to DSMC results using two different particle interaction models: Maxwell molecules and hard spheres. The VSS collision modelling of the DSMC method requires two parameters, the viscosity exponent $s^{(V S S)}$ and an additional parameter $\alpha^{(V S S)}$. For hard spheres these parameter are given by $s^{(V S S)}=0.5$ and $\alpha^{(V S S)}=1.0$, while for Maxwell molecules they are $s^{(V S S)}=1.0$ and $\alpha^{(V S S)}=2.26$, see Bird (1998). Since the DSMC code uses physical units we need to fix the mean free path of the upstream region $\lambda_{0}$ as length scale. We used

$$
\lambda_{0}^{(H S)}=0.00162 \mathrm{~m}, \quad \lambda_{0}^{(M M)}=0.001 \mathrm{~m}
$$

for hard spheres and Maxwell molecules, respectively, which corresponds to our definition (3.3) and also reproduces the shock thickness results of Pham-Van-Diep et al. (1991).

In the following figures we compare the profiles of density and heat flux. The heat flux in a shock wave follows solely from the temperature and velocity via relation (3.12). Hence, its profile gives an impression of the quality of the combined temperature and velocity profiles. The soliton-like shape of the heat flux also helps to give a more significant idea of the quality of the structure. Since it is a higher moment the heat flux is more difficult to match than the stress. We do not show the profiles of velocity, temperature and stress in the following. The density is normalized 

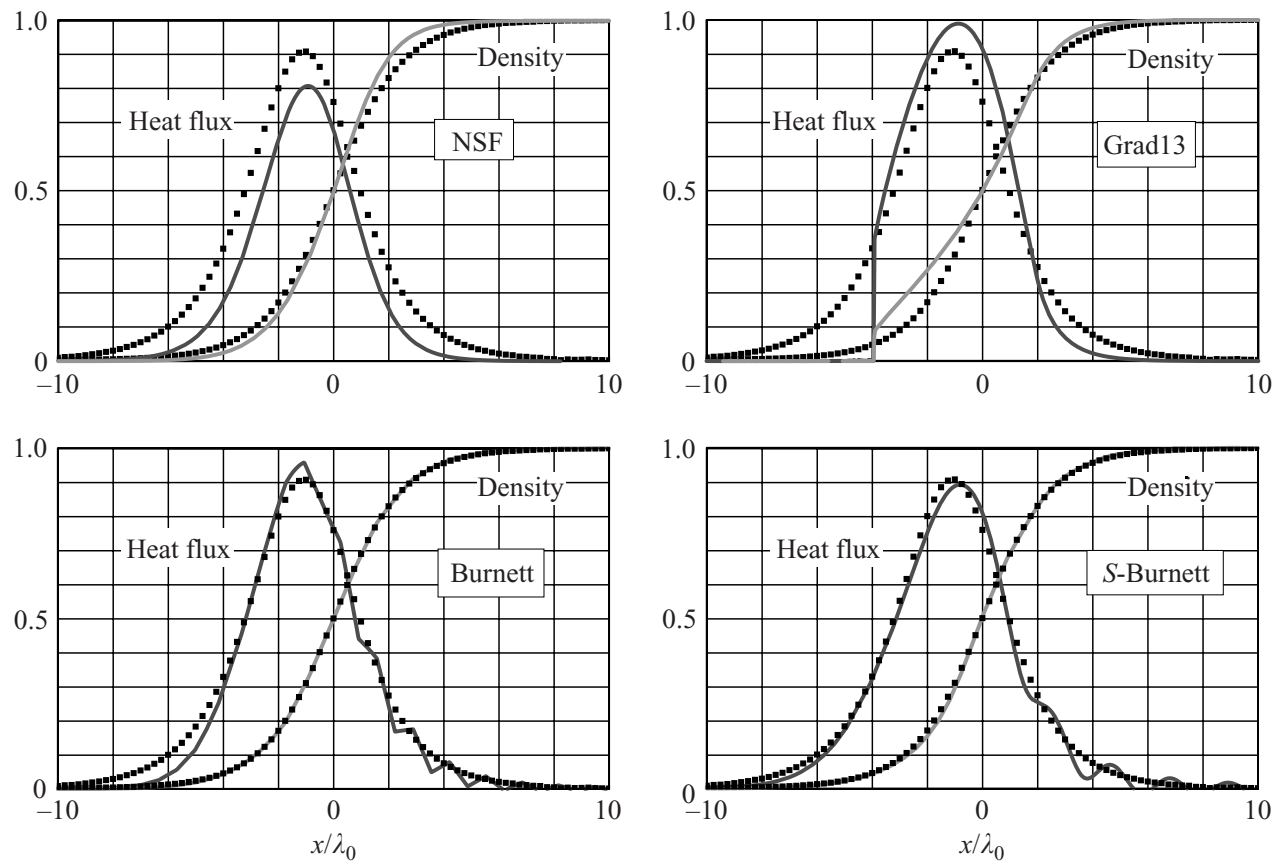

FiguRE 4. Shock structure solutions of the Navier-Stokes-Fourier system, the classical 13-moment-case of Grad and Burnett and super-Burnett equations for Maxwell molecules at Mach number $M_{0}=2$ and $s=1$ (solid lines). Both Burnett results exhibit non-physical oscillations in the downstream region. The squares represent the DSMC solution.

to give values between zero and unity for each Mach number. Similarly, the heat flux is normalized such that the DSMC result gives a maximal heat flux of 0.9.

\subsection{Maxwell molecules}

Before we present the results of the regularized 13-moment equations we discuss briefly the failure of the classical theories and the standard Burnett models. Figure 4 shows the density and heat flux profiles of a $M_{0}=2$ shock calculated with the NSF and Grad's 13-moment systems as well as with the Burnett and super-Burnett equations. The NSF results simply mismatches the profile, while the Grad 13 solution shows a strong subshock in addition. Burnett and super-Burnett solutions are spoiled by oscillations at the back of the shock.

In the Burnett case the oscillations arise if the length of a grid cell is below half of the mean free path. This corresponds to the result of the linear analysis which predicts spatial instabilities. It also explains the appearance of the oscillations in the downstream region, because the mean free path is smaller in that region. Since the oscillations maintain a wavelength corresponding to the length of a grid cell, high-resolution calculations are impossible. The super-Burnett result shows the same behaviour; however the oscillation wavelength is a multiple of the length of a grid cell. Still, the oscillations increase with grid refinement and convergence cannot be established.

The oscillations of both models, Burnett and super-Burnett, increase for shocks with higher Mach number and are also present for other values of the viscosity exponent. Hence, for the description of shock structures the Burnett-equations and super-Burnett-equations have to be rejected. The augmented Burnett equations are 

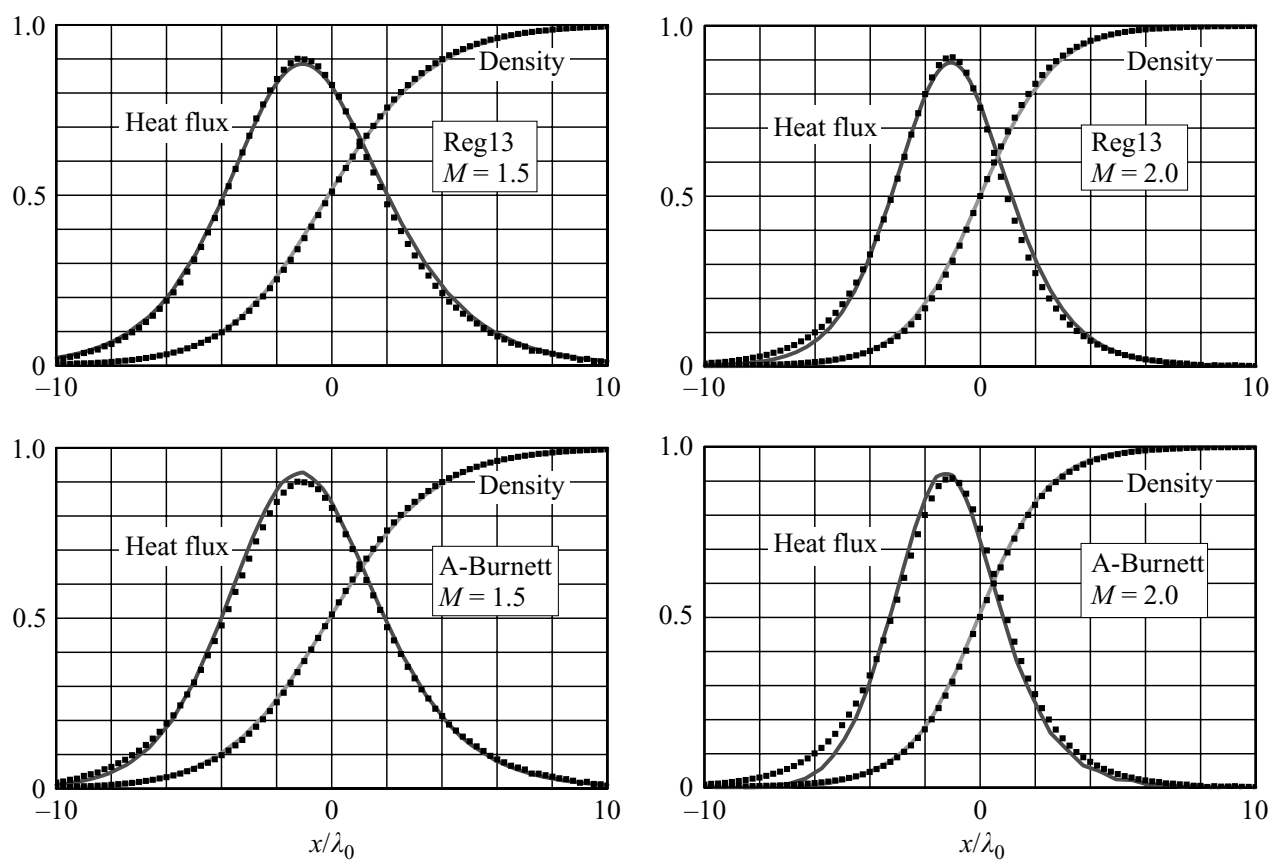

FIGURE 5. Shock structures in a gas of Maxwell molecules with $s=1$ and Mach numbers $M_{0}=1.5$ and $M_{0}=2.0$. Solid lines the upper row show the solution of the regularized 13-moment equations, and in the lower row the result of the augmented Burnett equations. The squares correspond to the DSMC solution.

stable in time but not in space. Since, we formulate the shock structures calculation as a boundary value problem the spatial stability is crucial. $\dagger$ In the following we will show several results of the augmented Burnett equations which are obtained on a coarse grid with $\Delta x \approx 0.5 \lambda_{0}$. On these grids the results show only small oscillations.

In figure 5 the shock structures for the Mach numbers $M_{0}=1.5$ and $M_{0}=2$ calculated with the R13 and augmented Burnett (A-Burnett) equations are displayed together with the DSMC result. The shape of the heat flux is captured very well by the R13 result, while the augmented Burnett equations seem to have difficulties matching the maximum value and the upstream relaxation. Note that the results for the density profile exhibit no visible differences and both match the DSMC result very nicely. The deviations from the DSMC solutions become more pronounced for higher Mach numbers. The plots in figure 6 show the results for $M_{0}=3$ and $M_{0}=4$. The R13 results starts to deviate from the DSMC solution in the upstream part. In the tail of the A-Burnett profiles small oscillations are present indicating the onset of instability.

From the figures presented we may conclude that the results of the R13 system for Maxwell molecules agree better with DSMC results than the solutions of the augmented Burnett equations. For higher Mach numbers the regularized 13-moment equations as well as the augmented Burnett system deviate even more from the DSMC

$\dagger$ In Zhong et al. (1991,1993) shock structures of the augmented Burnett equations are successfully calculated by use of a time-dependent method. 

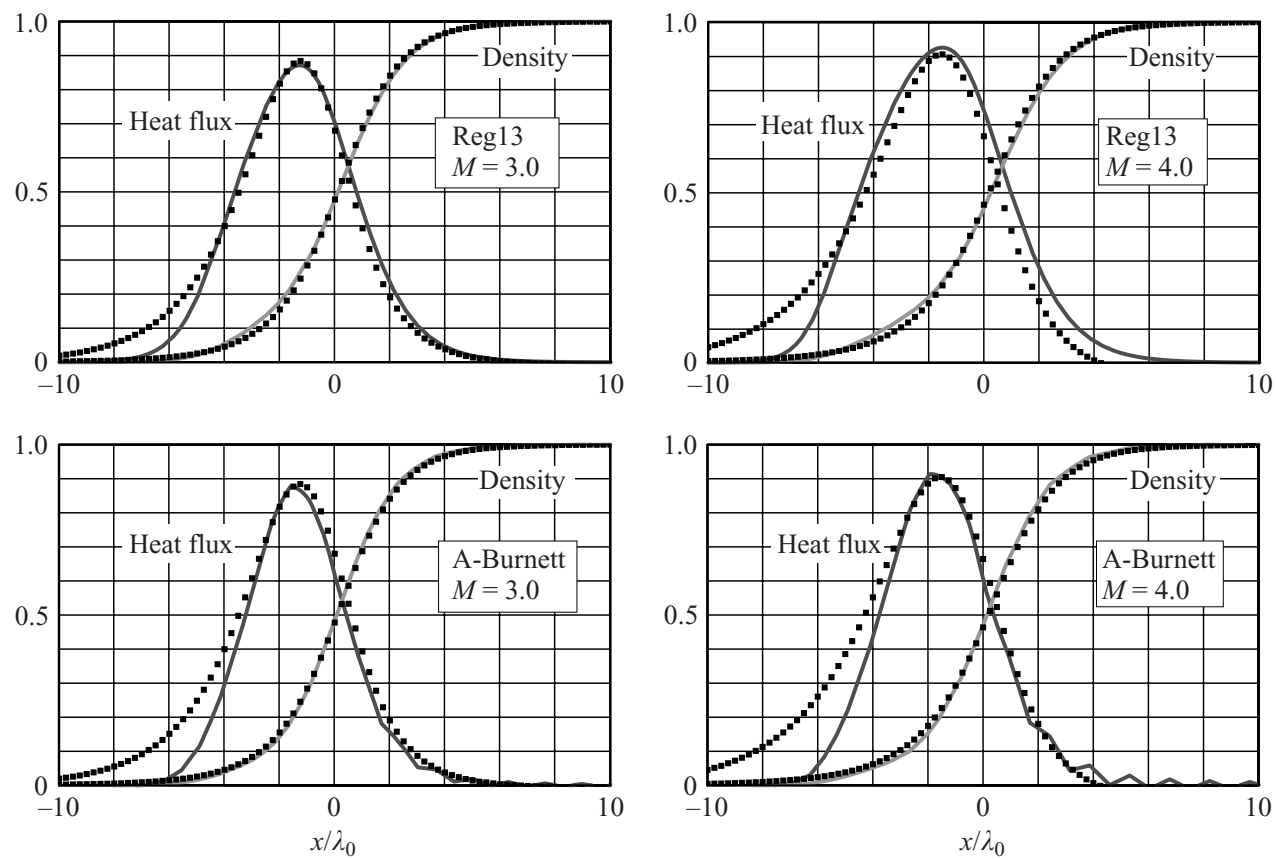

Figure 6. As figure 5 but with Mach numbers $M_{0}=3$ and $M_{0}=4$. Note the small oscillations in the result of the augmented Burnett equations.

result and the theories are no longer applicable, if quantitative features need to be captured.

\subsection{Hard spheres}

In this section we turn to the results for hard spheres, i.e. a viscosity exponent $s=0.5$. The original derivation of the regularized 13 -moment equations is based on integrals of the Boltzmann collision operator for Maxwell molecules, see Struchtrup $\&$ Torrilhon (2003). The ad hoc extension to more general particle interactions via the viscosity exponent (2.6) must be viewed as a first approximation. Indeed, in Struchtrup (2004b) it was shown that the full regularized 13-moment-system for hard-spheres must include the hard-sphere collision integrals of higher moments. An analogous statement applies to the Burnett models. As they are used here, the equations correspond to the first-order approximations of the collision integrals in terms of Sonine polynomials, see Chapman \& Cowling (1970, Chap. 15). The exact computation of the (Burnett) coefficients requires higher-order approximations. Note, however, that even for this simple extension, the change in the value of $s$ changes the coefficients in (2.9) and (2.10) due to differentiation of the viscosity during the derivation.

To give an impression of the performance of the hard-sphere modelling via (2.6) we present the shock structures for a hard-sphere gas for Mach numbers $M_{0}=$ 1.5, 2.0, 3.0, 4.0 in figures 7 and 8. As in the previous section the results of the continuum theories (solid lines) are compared to the DSMC solution (squares).

The regularized 13-moment equations have difficulties in describing the upstream relaxation while the tails of the structures are matched almost perfectly. Note that the upstream part of a shock is also more difficult to capture since it is the more rarefied region with larger mean free path. The solutions of the augmented Burnett equations 

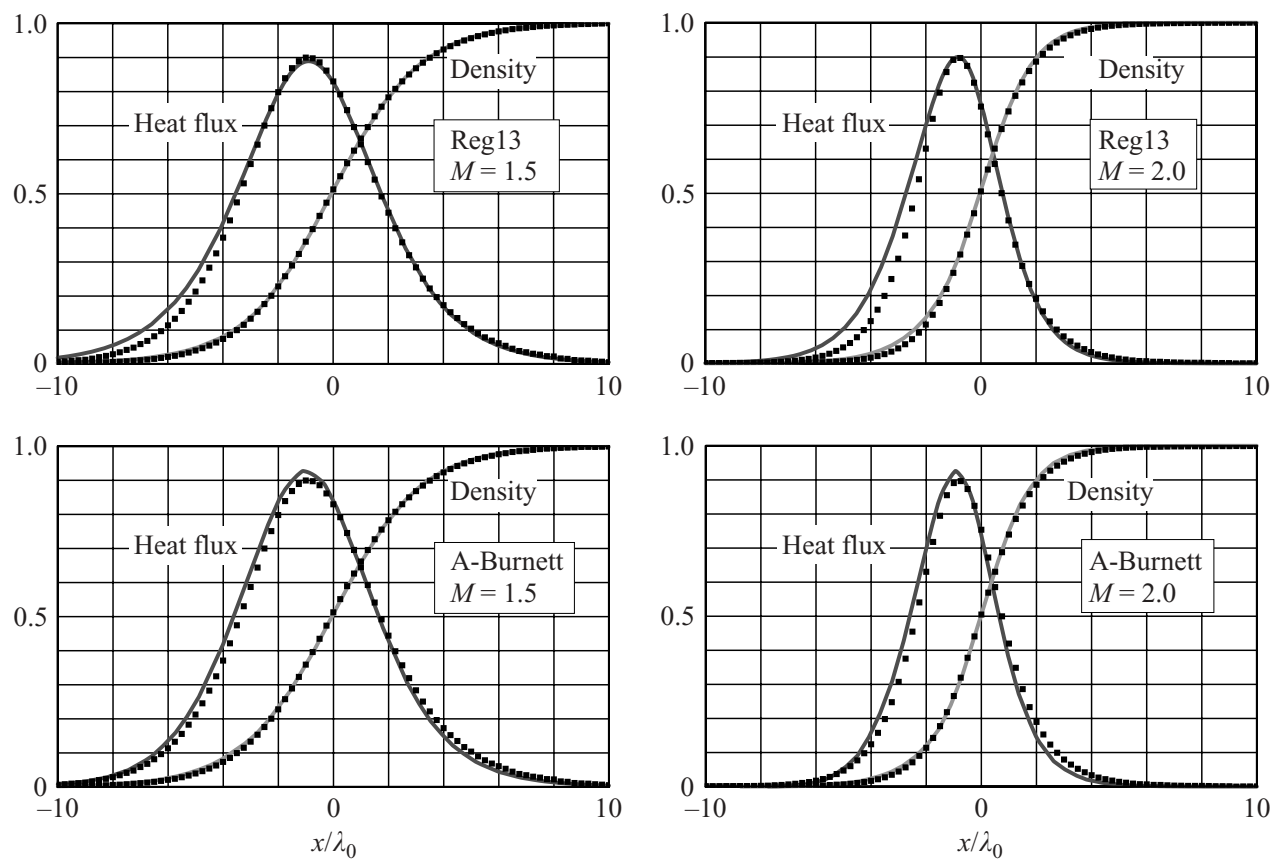

FIGURE 7. Shock structures in a gas of hard spheres with $s=0.5$ and Mach numbers $M_{0}=1.5$ and $M_{0}=2.0$. The solid lines show the results of the regularized 13-moment equations (upper row) and the augmented Burnett equations (lower row). The squares correspond to the DSMC solution.
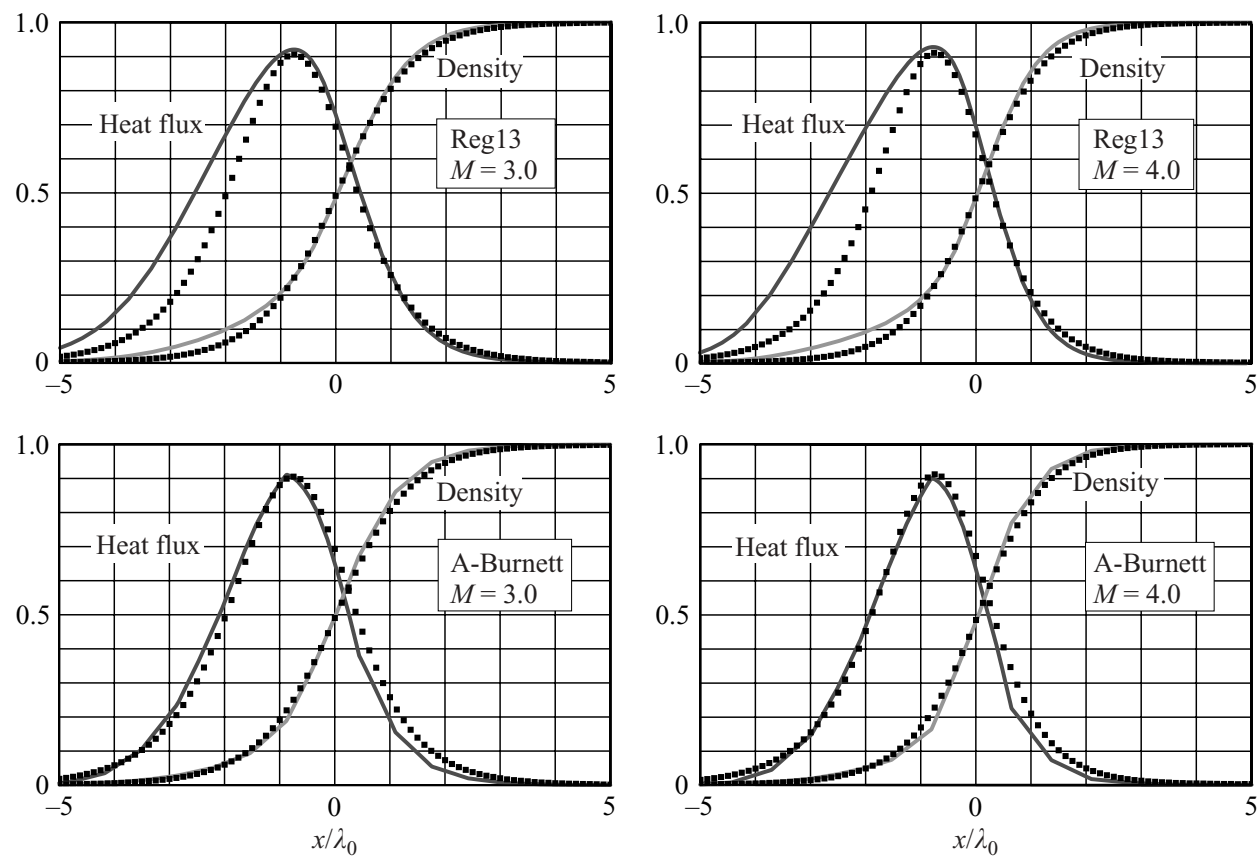

Figure 8 . Same as figure 7 but for Mach numbers $M_{0}=3$ and $M_{0}=4$. 


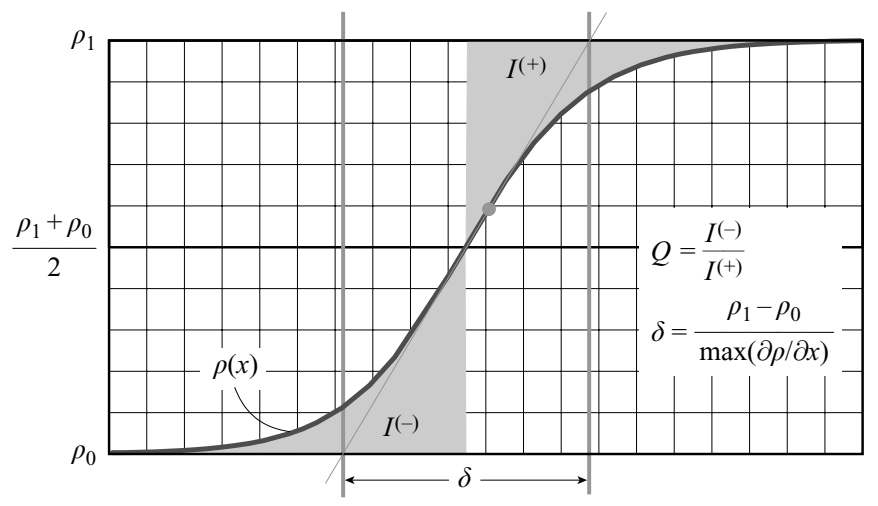

FiguRE 9. Definition of shock thickness and shock asymmetry for the density profile of a steady shock wave: The dot marks the position of the steepest gradient which is linearly extrapolated to give the shock thickness, $\delta$. The shock asymmetry, $Q$, follows from the ratio of the two integrals (shaded regions) of the upper and lower half of the profile.

fall short of the tail of the structure $\left(M_{0} \geqslant 3\right)$. The sharp-edged shape of the result of the A-Burnett equations is due to the coarse resolution.

Altogether, the A-Burnett system seems to give better results for hard spheres than for Maxwell molecules. Note, however, that the coarse resolution and spatial instability of the equations calls their reliability into question. On the other hand, the results of the regularized 13-moment equations also need improvements for gases of hard spheres.

\section{Shock thickness and asymmetry}

In the following subsections, we will present shock thickness and shock asymmetry results obtained with the regularized 13-moment equations and the various Burnett models as well as the Navier-Stokes-Fourier system and the classical Grad equations. The results will be compared to DSMC results and experimental data. The oscillatory behaviour of the Burnett and super-Burnett results could be ignored, since only the density profile is considered which is not much affected by the oscillations.

\subsection{Characterization of shock structures}

Instead of comparing complete profiles, each shock profile is often assigned only a few parameters which characterize its most important properties, thus reducing the amount of complexity. The graph in figure 9 demonstrates the definition of the two most often used profile characteristics, thickness and asymmetry.

The so-called shock thickness (see e.g. Gilberg \& Paolucci 1953; Schmidt 1969; Alsmeyer 1976) is defined as

$$
\delta=\frac{\rho_{1}-\rho_{0}}{\max (\partial \rho / \partial x)}
$$

and assumes a linear connection between the density values before and behind the shock and a slope corresponding to the steepest density gradient. The shock thickness starts with infinite values for shocks with $M_{0} \rightarrow 1$, approaches a minimum of several mean free paths at Mach numbers around 3 to 5 and finally tends slowly to infinity again as $M_{0} \rightarrow \infty$. This non-monotone behaviour represents an interplay between 

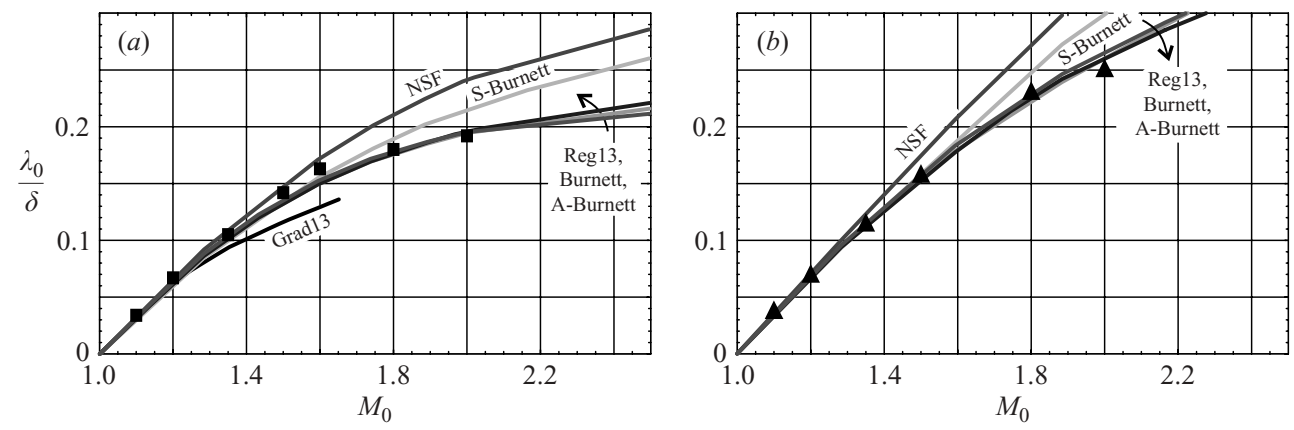

FIGURE 10. Shock thickness results for Maxwell molecules, $s=1,(a)$ and hard spheres, $s=0.5,(b)$ calculated with the regularized 13-moment equations, Burnett, super-Burnett and augmented Burnett, Navier-Stokes-Fourier and Grad13. The symbols represent the results of DSMC simulations.

steepening nonlinearity and smoothing dissipation. Usually the shock thickness is related to the mean free path in front of the shock $\bar{\lambda}_{0}$ and the inverse ratio $\bar{\lambda}_{0} / \delta$ is plotted.

The shock asymmetry (introduced in Schmidt 1999) gives more information about the actual shape of the profile than the shock thickness. The asymmetry is defined as

$$
Q=\frac{\int_{-\infty}^{x^{\star}}\left(\rho(x)-\rho_{0}\right) \mathrm{d} x}{\int_{x^{\star}}^{\infty}\left(\rho_{1}-\rho(x)\right) \mathrm{d} x}
$$

where the position $x^{\star}$ is determined by the relation $\rho\left(x^{\star}\right)=\frac{1}{2}\left(\rho_{0}+\rho_{1}\right)$. For realistic shock waves the asymmetry is close to unity, i.e. the measured profiles are rather symmetric. Typical values lie between 0.8 and 1.2, see Alsmeyer (1976). An asymmetry value $Q<1$ indicates a relatively slower relaxation behind the shock.

Shock thickness and asymmetry are usually defined by means of the density field for reasons of experimental feasibility.

\subsection{Shock thickness}

In order to evaluate the low-Mach-number range, shock structures have been calculated for Mach numbers $M_{0}=1.2,1.25,1.35,1.5,1.651,1.8,2.0$ and 2.5 with viscosity exponent $s=0.5$ and $s=1$, i.e. for hard-spheres and Maxwell molecules. The shock thickness values obtained with these calculations are linearly interpolated in order to obtain continuous curves. The results for the shock thickness are compared with DSMC calculations from Pham-Van-Diep et al. (1991) which also have been reproduced by our DSMC simulations.

Figure 10 shows the theoretical curves for the inverse shock thickness, see (6.1), of the various continuum models together with the DSMC results as symbols. Here, plot $(a)$ correspond to Maxwell molecules and plot $(b)$ to a gas of hard spheres. Both plots use the same scales for the axes. The comparison of the curves shows the clear advantage of the higher-order models like R13 or Burnett over NSF and Grad 13. The super-Burnett results, however, do not show a better correspondence than the lower-order Burnett equations. The curves for R13, Burnett and A-Burnett follow the symbols quite accurately. Note that the DSMC calculations are subject to 


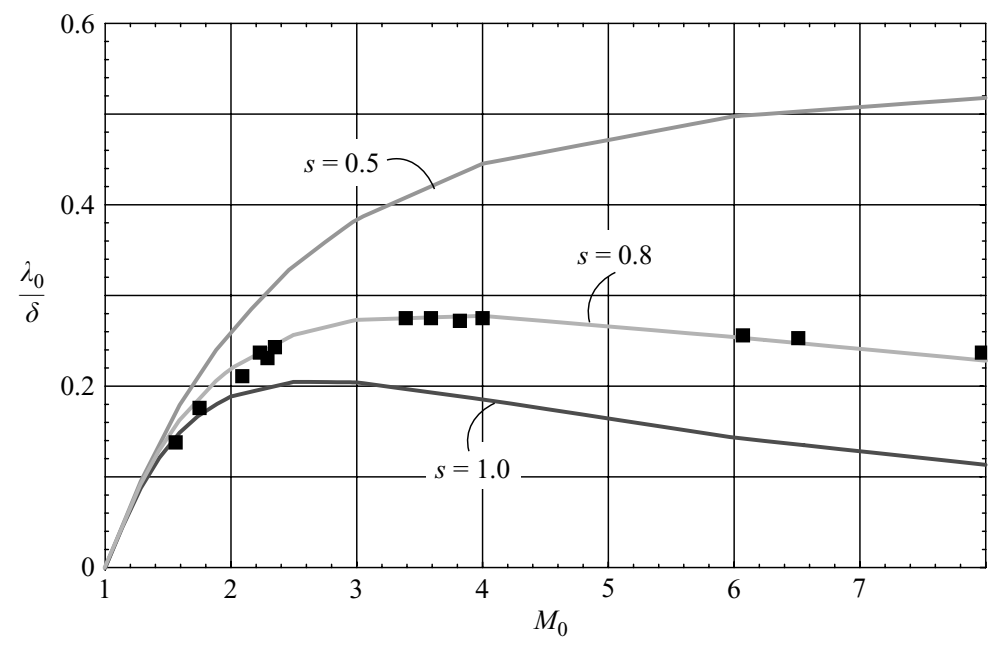

FIGURE 11. Comparison of inverse shock thickness results of regularized 13-moment equations with measurements for argon (squares, $s \approx 0.8$ ). The curve of the augmented Burnett equations with $s=0.8$ shows a similar agreement.

statistical errors, so that slight differences between DSMC results and predictions of the continuum models are acceptable.

Additionally, shock structures have been calculated for Mach numbers $M_{0}=$ 3.0, 4.0, 6.0 and 8.0. As has been mentioned in $\S 4.1$ the profile begins to lose its validity, in particular because kinks arise. Nevertheless, the profile stays smooth and the shock thickness is well defined. In figure 11 the results of the R13 system are compared to the measurements of the inverse shock thickness in argon given in Alsmeyer (1976) (also Schmidt 1969). The value $s=0.8$ for the viscosity exponent in the regularized 13-moment equations yields a striking agreement with the experimental data. This value of the viscosity exponent of argon is also given in the textbooks by Chapman \& Cowling (1970) and Bird (1998). We remark that the results of the augmented Burnett equations with $s=0.8$ lead to a similar agreement, while the Navier-Stokes-Fourier system lies far away.

To some extent the good agreement of the shock thickness for high Mach number should be seen as a lucky coincidence. The detailed shape of the profiles for high Mach numbers obviously does not match the profiles shown in Alsmeyer (1976). It becomes evident that the single parameter $\delta$ cannot reflect the complete profile so that the agreement with shock thickness measurements does not imply a reliable description of the complete profile. Nevertheless, the information that $\delta$ does reflect - a mean thickness - is predicted by the R13 equations very accurately even for high Mach numbers.

\subsection{Shock asymmetry}

The shock asymmetry (6.2) is another characteristic measure of the shock profile. In Pham-van-Diep et al. (1991) shock asymmetry results for Maxwell molecules and hard spheres obtained by DSMC simulations are presented, which are shown in figure 12. Plot $(a)$ shows the results for gas of Maxwell molecules and plot $(b)$ results for hard spheres. Additionally, the figure includes the theoretical curves of the NSF theory, Grad's 13-moment case, the R13 solutions and the Burnett models. None of the curves exhibit reasonable agreement with the DSMC results. 

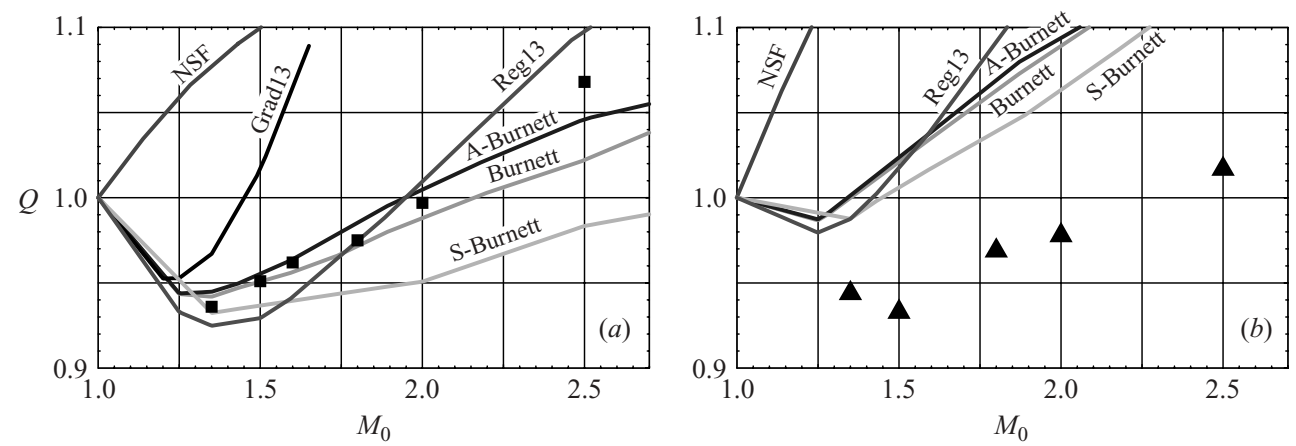

FIGURE 12. Shock asymmetry result for Maxwell molecules, $s=1,(a)$ and hard spheres, $s=0.5$, (b) calculated with the regularized 13-moment equations, Burnett, super-Burnett, augmented Burnett, and Navier-Stokes-Fourier as well as Grad13. The symbols represent the results of DSMC simulations.

First of all, the results of NSF indicate a qualitative failure. Both curves predict an asymmetry $Q>1$ from the very beginning (i.e. already for small Mach numbers), which is in clear contradiction not only to DSMC simulations but also to measurements, see e.g. Alsmeyer (1976). The R13 solution for $s=1$ matches the DSMC results around $M_{0}=2$, while the Burnett and A-Burnett equations give a reasonable agreement below $M_{0}=2$. The asymmetry results of the classical 13-moment-system of Grad and of the super-Burnett equations lie far away.

In the plot for hard-spheres all curves of the continuum models are a long way from the DSMC results. For hard-spheres all curves give asymmetry results larger than the results for a Maxwell gas. In contrast, the DSMC results tend to predict a smaller asymmetry. This is a strong contradiction. However, the continuum modelling of the hard-sphere gas via the viscosity (2.6) is only a first approximation. A more careful investigation with better approximations is needed, and planned for the future.

\section{Positivity of the distribution function}

Close to and at equilibrium the distribution function for the microscopic velocity of the gas particles is given by the Maxwellian distribution

$$
f_{M}=\frac{\rho}{\sqrt{2 \pi T}^{3}} \exp \left(-C^{2} / 2 T\right),
$$

where density $\rho$, temperature $T$ and characteristic velocity of the particles $C_{i}$ are dimensionless according to (3.1). In non-equilibrium flows, especially in shock waves, the distribution function deviates markedly from the Maxwellian (7.1). Since the system of the regularized 13-moment equations still consider Grad's 13 moments as variables, we assume Grad's 13-moment distribution function given in Grad (1958) or Müller \& Ruggeri (1998) as an approximation to the real distribution function in the particular flow. In the one-dimensional formulation, Grad's distribution is

$$
f_{13}=f_{M}\left(1+\frac{\sigma}{4 \rho T^{2}}\left(3 C_{x}^{2}-C^{2}\right)-\frac{q}{\rho T^{2}} C_{x}\left(1-\frac{1}{5 T} C^{2}\right)\right)
$$

where $C$ is, as in (7.1), the norm of the characteristic velocity and $C_{x}$ is its component normal to the shock. Both $C$ and $C_{x}$ are given by the norm of the absolute velocity $c$, 


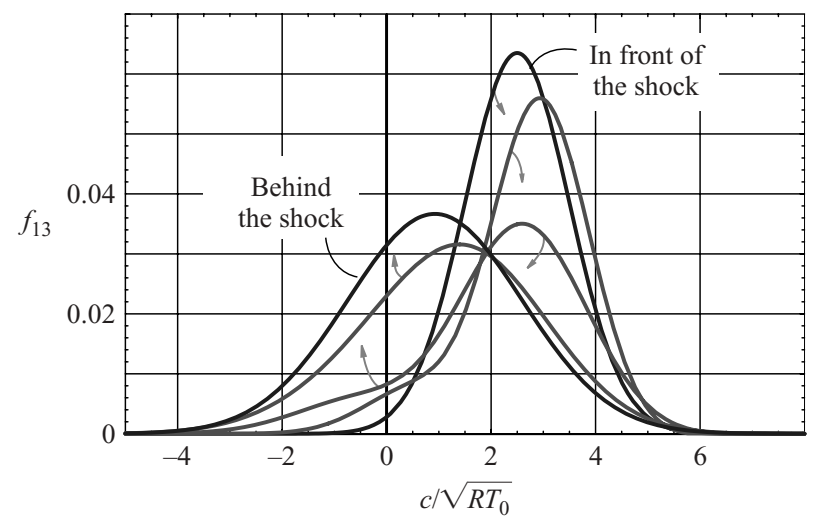

FIGURE 13. Sketch of the distribution function in normal direction $(\vartheta=0)$ in front and behind the shock as well as at three positions inside the shock. The Mach number is $M_{0}=2.5$. Inside the shock the distribution functions becomes a bimodal distribution.

the flow velocity $v$ and an angle of direction $\vartheta$ via the relations

$$
\begin{aligned}
& C_{x}=c \cos \vartheta-v, \\
& C^{2}=c^{2}-2 c v \cos \vartheta+v^{2} .
\end{aligned}
$$

The angle $\vartheta=0$ represents the direction normal to the shock. Thus, Grad's distribution $f_{13}(c, \vartheta \mid \rho, v, T, \sigma, q)$ can be considered as a function of the flow quantities and $c$ and $\vartheta$.

With the results from the numerical calculations of the shock profiles we can plot the distribution function by virtue of (7.2) at any position in the shock. Figure 13 shows the distribution function at three positions inside a Mach 2.5 shock $(s=1)$ together with the initial and final Maxwell distributions. The shock transforms a right-positioned and narrow distribution into a more leftward and flat one, which indicates the transition from high velocity and low temperature to low velocity and high temperature. Inside the shock, the distribution function exhibits an asymmetric shoulder representing the onset of bimodality which is also observed in DSMC simulations (Bird 1998).

Grad's distribution is known to admit non-physical negative values, since the polynomial expression in (7.2) may change its sign. Indeed, in figure 13 negative values for $f_{13}$ are present but these occur only for large velocities $(c>6)$ where the Maxwellian in (7.2) already guarantees that the function $f_{13}$ is almost zero (at $c=6, f_{13}$ is about $10^{-4}$ and decreases for larger $c$ ). This means that $f_{13}$ is positive where its absolute value is significantly different from zero. We conclude that the results depicted in figure 13 indeed refer to a valid description of a Mach 2.5 shock.

However, the threat of negative values becomes more significant for higher Mach numbers. Figure 14 displays distribution functions inside shocks of various Mach numbers. Each distribution function is taken at the position $x^{\star}$, where $T\left(x^{\star}\right)=$ $\frac{1}{2}\left(T_{0}+T_{1}\right)$, at which the deviation from a Maxwellian was found to be most pronounced. For a shock with Mach number $M_{0}=4$ the distribution function becomes significantly negative in a relevant range. We propose that this negativity indicates the loss of validity of our regularized 13-moment equations. 


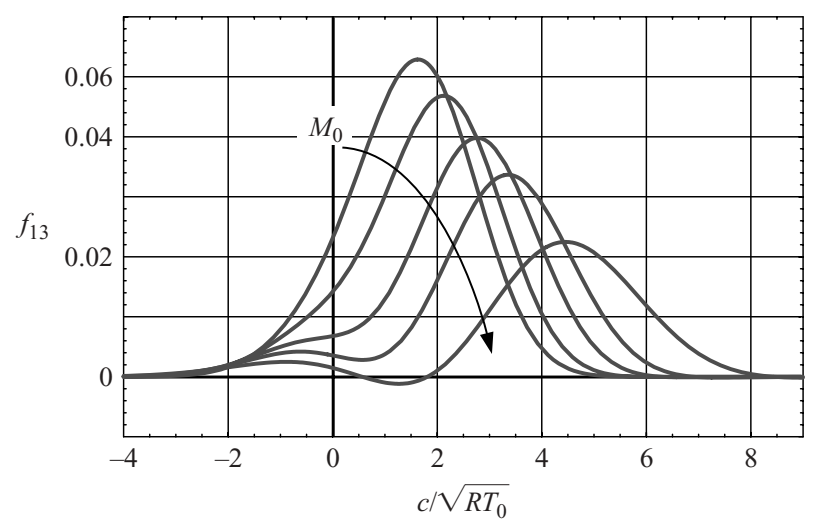

FIGURE 14. Distribution functions normal to the shock $(\vartheta=0)$ inside shock profiles with increasing Mach numbers $M_{0}=1.651,2.0,2.5,3.0,4.0$. The distribution function is taken at the position $x^{\star}$ where $T\left(x^{\star}\right)=\frac{1}{2}\left(T_{0}+T_{1}\right)$.

\section{Conclusion}

The new regularized 13-moment equations (R13), derived in Struchtrup \& Torrilhon (2003), contain not only the Burnett equations, but also the nonlinear super-Burnett equations asymptotically for small Knudsen numbers. This has been shown by comparing the nonlinear expansion of the one-dimensional equations with the results found in the literature. However, in contrast to the Burnett models the regularized 13moment-system is stable. We also compared the new system to the existing models of the augmented Burnett equations of Zhong et al. (1991) and to the regularized Burnett system of Jin \& Slemrod (2001). The main differences of the R13 equations are:

(i) They are stable with respect to time and space, while the augmented Burnett equations are only stable in time.

(ii) They exhibit the proper tensorial structure induced by the transfer equations of the Boltzmann equation, while both of the other models do not.

(iii) They follow rigorously from the Boltzmann equation, while both of the other models do not.

Finally we presented shock structure solutions of the regularized 13-moment equations for Maxwell molecules and hard spheres. The paper demonstrated that the R13 equations yield smooth shock structures over a wide range of Mach numbers. Hence, it resolves the subshock problem in Grad's original equations. Furthermore, the calculated profiles have been compared to DSMC solutions as well as to the solutions of the augmented Burnett equations. The agreement with DSMC results is better for the R13 equations than the augmented Burnett equations in the case of Maxwell molecules and vice versa in the case of hard spheres. However, the results of the augmented Burnett equations suffer from oscillations and are restricted to coarse grid resolutions. From the comparison with DSMC results and from an analysis of the positivity of Grad's distribution function inside the shock we concluded that quantitative features of the shock will only be captured up to a Mach number $M_{0} \approx 3.0$. Still, the theory will be useful for high-Mach-number flow if quantitative features are less important.

This research was supported by the Natural Sciences and Engineering Research Council (NSERC). The authors are also grateful to A. Schuetze (Univ. Victoria) for managing several DSMC calculations. 


\section{REFERENCES}

Agarwal, R. K., Yun, K. Y. \& Balakrishnan, R. 2001 Beyond Navier-Stokes: Burnett equations for flows in the continuum-transition regime. Phys. Fluids 13, 3061-3085, and Erratum: 14, (2002), 1818.

AlsmeYer, H. 1976 Density profiles in argon and nitrogen shock waves measured by the absorbtion of an electron beam. J. Fluid Mech. 74, 497-573.

Au, J. D. 2001 Lösung nichtlinearer Probleme in der Erweiterten Thermodynamik, Dissertation, TU Berlin.

Au, J. D., Torrilhon, M. \& Weiss, W. 2001 The shock tube study in extended thermodynamics. Phys. Fluids 13, 2423-2432.

Bhatnagar, P. L., Gross, E. P. \& KrooK, M. 1954 A model for collision processes in gases. I. Small amplitude processes in charged and neutral one-component systems. Phys. Rev. 94, 511-525.

BIRD, G. A. 1998 Molecular Gas Dynamics and the Direct Simulation of Gas Flows, 2nd edn. Oxford University Press.

Bobylev, A. V. 1982 The Chapman-Enskog and Grad methods for solving the Boltzmann equation. Sov. Phys. Dokl. 27, 29-31.

Chapman, S. \& Cowling, T. G. 1970 The Mathematical Theory of Non-Uniform Gases. Cambridge University Press.

Fiscko, K. A. \& Chapman, D. R. 1989 Comparison of Burnett, Super-Burnett and Monte Carlo solutions for hypersonic shock structure. In Proc. 16th Symp. on Rarefied Gasdynamics, AIAA, Washington, pp. 374-395.

Gilbarg, D. \& PAOLuCCI, D. 1953 The structure of shock waves in the continuum theory of fluids. J. Rat. Mech. Anal. 2, 617-642.

Grad, H. 1949 On the kinetic theory of rarefied gases. Commun. Pure Appl. Maths 2, 331-407.

Grad, H. 1952 The profile of a steady plane shock wave. Commun. Pure Appl. Maths 5, 257-300.

Grad, H. 1958 Principles of the kinetic theory of gases. In Handbuch der Physik, vol. 12 (ed. S. Flügge), pp. 205-251. Springer.

Holway, L. H. 1966 New statistical models for kinetic theory: methods of construction. Phys. Fluids 9, $1658-1673$.

Jin, S. \& Slemrod, M. 2001 Regularization of the Burnett equations via relaxation. J. Statist. Phys. 103(5-6), 1009-1033.

Jin, S., Pareschi, L. \& Slemrod, M. 2002 A relaxation scheme for solving the Boltzmann equation based on the Chapman-Enskog expansion. Acta Math. Appl. Sinica (English Series) 18, 37-62.

Karlin, I. V., Gorban, A. N., Dukek, G. \& Nonnenmacher, T. F. 1998 Dynamic correction to moment approximations. Phys. Rev. E 57(2), 1668-1672.

Karlin, I. V. \& Gorban, A. N. 2002 Hydrodynamics from Grad's equations: What can we learn from exact solutions? Ann. Phys. Berlin 11(10-11), 783-833.

Kogan, M. N. 1969 Rarefied Gas Dynamics. Plenum.

MüLler, I. \& Ruggeri, T. 1998 Rational Extended Thermodynamics, 2nd edn. Springer Tracts in Natural Philosophy, vol. 37.

NowaK, U. \& Weimann, L. 1990 A family of Newton codes for systems of highly nonlinear equations - algorithms, implementation, applications. Zuse Institute Berlin, Tech. Rep. TR 90-10.

Pham-Van-Diep, G. C., Erwin, D. A. \& Muntz, E. P. 1991 Testing continuum descriptions of low-Mach-number shock structures. J. Fluid Mech. 232, 403-413.

Reinecke, S. \& Kremer, G. M. 1990 Method of moments of Grad. Phys. Rev. A 42, 815-820.

Reinecke, S. \& Kremer, G. M. 1996 Burnett's equations from a $(13+9 \mathrm{~N})$-field theory. Continuum Mech. Thermodyn. 8, 121-130.

RugGeri, T. 1993 Breakdown of shock wave structure solutions. Phys. Rev. E 47, 4135-4140.

ShavaliYev, M. S. 1993 Super-Burnett corrections to the stress tensor and the heat flux in a gas of Maxwellian molecules. J. Appl. Maths Mech. 57(3), 573-576.

Schmid, B. 1969 Electron beam density measurements in shock waves in argon. J. Fluid Mech. 39, 361-366.

Struchtrup, H. 2004a Some remarks on the equations of Burnett and Grad. In IMA Vol. Math. Appl. 735. Springer. 
Struchtrup, H. $2004 b$ Derivation of 13 moment equations for rarefied gas flows to second order accuracy for arbitrary interaction potentials. Multiscale Model. Sim. (accepted).

Struchtrup, H. \& Torrilhon, M. 2003 Regularization of Grad's 13-moment equations: derivation and linear analysis. Phys. Fluids 15, 2668-2680.

Torrilhon, M. 2000 Characteristic waves and dissipation in the 13-moment-case. Continuum Mech. Therm. 12, 289-301.

Uribe, F. J., Velasco, R. M. \& Garcia-Colin, L. S. 1998 Burnett description of strong shock waves. Phys. Rev. Lett. 81, 2044-2047.

Wang Chang, C. S. 1970 On the theory of the thickness of weak shock waves. In Studies in Statistical Mechanics V, pp. 27-42. North Holland.

WeIss, W. 1995 Continuous shock structures in extended thermodynamics. Phys. Rev. E 52, 57605763.

Zheng, Y. \& Struchtrup, H. 2003 Burnett equations for the ellipsoidal statistical BGK Model. Continuum Mech. Thermodyn (to appear).

Zhong, X., MacCormack, R. W. \& Chapman, D. R. 1991 Stabilization of the Burnett equations and applications to high-altitude hypersonic flows. AIAA Paper 91-0770.

Zhong, X., MacCormack, R. W. \& Chapman, D. R. 1993 Stabilization of the Burnett equations and applications to hypersonic flows. AIAA J. 31, 1036-1043. 